\title{
Major chemical and mineral components of profundal surface sediments in Minnesota lakes ${ }^{1}$
}

\author{
Walter E. Dean ${ }^{2}$
}

Department of Geology, Syracuse University, Syracuse, New York 13210

\section{Eville Gorham}

Department of Ecology and Behavioral Biology, University of Minnesota, St. Paul 55108

\begin{abstract}
Minnesota lakes, characterized primarily on the basis of water chemistry, can also bc classified according to the proportions of organic matter, $\mathrm{CaCO}_{\mathrm{i}}$, and clastic minerals in their profundal sediments. The northeastern (group 1) lakes are characterized by shallow to very deep basins in Precambrian crystalline rocks, active outlets, and dilute surface waters. Profundal sediments of these lakes contain no sedimentary calcium carbonate and can be subdivided on the basis of organic content. Highly organic sediments of group 1 lakes contain more than $30 \%$ organic matter (as loss on ignition), but most group I lake sediments contain less than this.

The central group 2 lakes occupy shallow to moderately deep basins in calcareous glacial till, have inactive outlets as a result of net evaporation, contain more concentrated salts than group 1 lakes, and commonly precipitate $\mathrm{CaCO}_{3}$ during the summer. These lakes can be subdivided on the basis of sedimentary $\mathrm{CaCO}_{3}$. Sediments of group 2 lakes with more than $30 \%$ organic material contain little or no $\mathrm{CaCO}_{3}$. Southern group 2 lake sediments are characterized by $10-30 \% \mathrm{CaCO}_{3}$ and $10-30 \%$ organic mattcr. Sediments of western group 2 lakes contain $30-50 \% \mathrm{CaCO}_{3}$ and about twice as much clastic as organic materials. Group 3 lakes occupy shallow depressions in calcareous, sulfur-rich glacial drift of the arid southwestern prairie region and contain even more concentrated salts than group 2 lakes. Sediments in these lakes are mainly clastic, averaging about $12 \%$ organic matter and $20 \% \mathrm{CaCO}_{3}$.

Variations in water and sediment chemistry, and in sediment mineralogy of these lakes are clearly related to an increase in climatic aridity and aquatic productivity from northeast to southwest. Differences in nature of the underlying glacial drift and in degree of erosion also affect the composition of the lake sediments.
\end{abstract}

Minnesota has more than 15,000 lakes in a landscape characterized by diverse bedrock and surficial geology (Fig. 1). It also exhibits marked climatic gradients. Annual prccipitation is $70-80 \mathrm{~cm}$ in the eastern half of the state and $50-70 \mathrm{~cm}$ in the western half (Ackroyd et al. 1967). Runoff exceeds $25 \mathrm{~cm}$ in northeastern Minnesota, $20 \mathrm{~cm}$ in the southeast, and falls to less than $5 \mathrm{~cm}$ in the west (Minn. Dep. Conserv. 1962). Mean annual temperatures range from $2{ }^{\circ} \mathrm{C}$ in the northeast to $8^{\circ} \mathrm{C}$ in the south (Baker and Strub 1965). According to Bright

\footnotetext{
${ }^{1}$ Contribution No. 135, Limnological Research Center, University of Minnesota. This work was supported by National Science Foundation grants GB-6018 and GB 18800 and the Graduate School, University of Minnesota.

${ }^{2}$ Present address: U.S. Geological Survey, Branch of Regional Geochemistry, Bldg. 53, Federal Center, Lakewood, Colorado 80225.
}

(1968), the balance of precipitation minus evaporation in the state is positive $(0-8 \mathrm{~cm})$ only in the northeast and is distinctly negative $(-15$ to $-30 \mathrm{~cm})$ in the west.

The chemistry of surface waters reflects the combined influences of these environmental variables, and on this basis Gorham et al. (in prep.) have divided Minnesota lakes into four distinct geographic groups (Fig. 2). The northeastern (group 1) lakes contain dilute bicarbonate waters (conductivity $<160 \mu \mathrm{mhos} \mathrm{cm}^{-1}$ at $25^{\circ} \mathrm{C}$ ) in shallow to relatively deep basins (up to $64 \mathrm{~m}$ ) in Precambrian crystalline rocks. In this heavily forested region precipitation exceeds evaporation, so that the northeastern lakes have active outlets throughout the year. A few group 1 lakes are also found elsewhere on coarse, noncalcareous drift. The central (group 2) lakes contain bicarbonate waters of intermcdiate concentration (conductiv- 

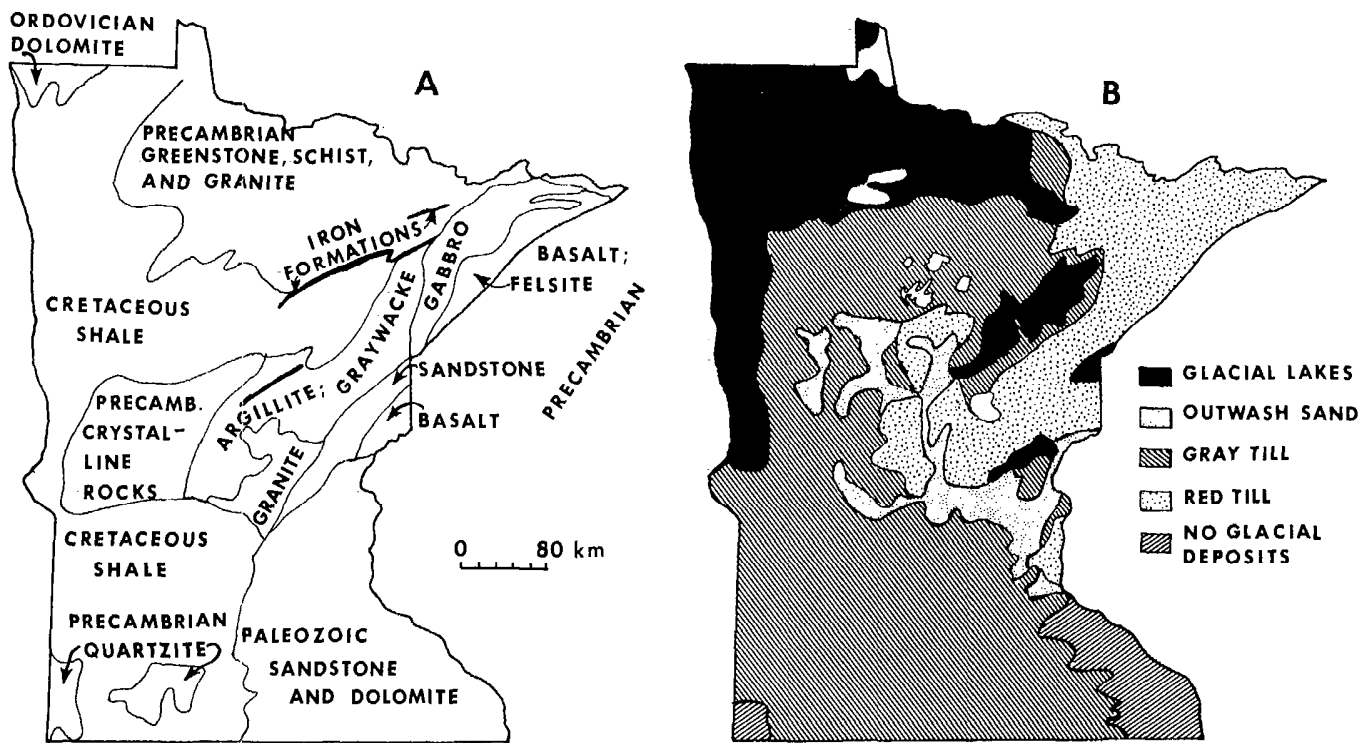

Fig. 1. Maps of bedrock geology (A) and major surficial deposits (B) (modified from Ackroyd et al. 1967 and Winter 1974).

ity $160-500 \mu \mathrm{mhos} \mathrm{cm}^{-1}$ at $25^{\circ} \mathrm{C}$ ) in shallow to moderately deep basins in calcarcous drift. The central lakes are found in both forest and prairie regions, generally have inactive outlets, and many (or most) of them precipitate carbonate biogenically during summer (Megard 1968). Group 3 lakes have high concentrations of bicarbonate and sulfate waters (conductivity $>500 \mu \mathrm{mhos} \mathrm{cm}^{-1}$ at $25^{\circ} \mathrm{C}$ ) and occupy shallow depressions in sulfur-bcaring glacial drift (cm. Winter 1974) in the southwestern prairic region. Group 4 consists of a very few lakes along the western border with high concentrations of sodium and sulfate (conductivity $>7,000 \mu \mathrm{mhos} \mathrm{cm}^{-1}$ at $25^{\circ} \mathrm{C}$ ); there are many more such lakes in the Dakotas (e.g. Mitten et al. 1968). Within Minnesota, concentrations of dissolved salts in lake water increases more than 1,000-fold from the northeastern lakes to those of the southwest-this gradient reflecting mainly the change from positive to negative balance between precipitation and evaporation along the same gradient (Bright 1968). The boundaries between groups are rclatively sharp, however, suggesting that surficial or bedrock geology, or both, are more important than climate in determining actual group boundaries.

Here we analyze the major components of profundal sediments from 46 lakes ( 14 group 1, 27 group 2, 4 group 3, and 1 group 4 ) in relation to environmental factors. The top $10 \mathrm{~cm}$ of profundal sediments from the deepest portions of the lakes were chosen for analysis in the belief that they would provide the best integration of allochthonous materials from the drainage basin and autochthonous materials produced within the lakc. Analyses of sediments along transects in scveral lakes demonstrated that excessive solution of sedimentary carbonate minerals in the deeper portions of some lakes is the most serious error in this assumption. This problem is discessed in a later section.

We are grateful to H. E. Wrighr, Jr., G. J. Brunskill, and R. Y. Anderson for helpful reviews of the manuscript. Additional suggestions, particularly regarding clay mineralogy, were provided by W. Parham.

\section{Methods}

Undisturbed samples of profundal sediments were collected with a Jenkin sampler 


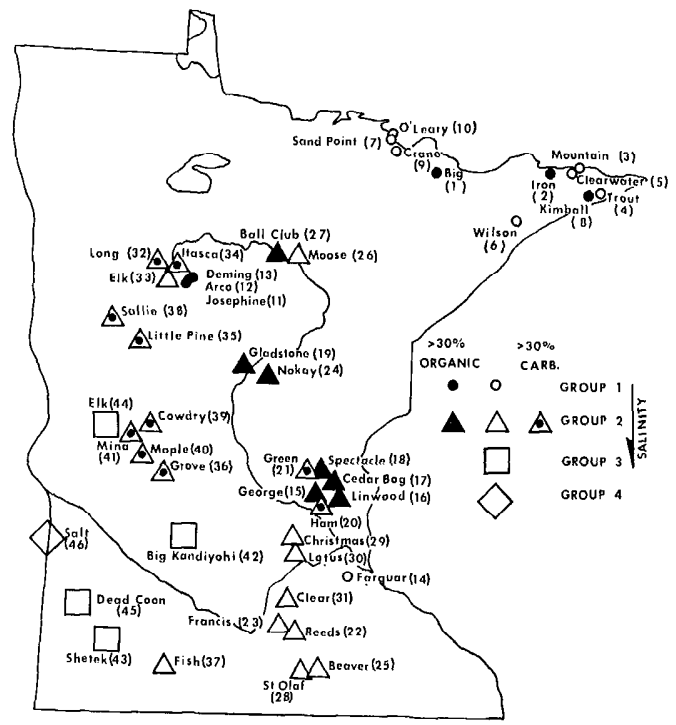

Fig. 2. Map of lakc location and profundal scdiment composition for all 46 lakes. Lakes are numbered in order of increasing salinity, used for reference in tables. Symbols reflect surface water salinity (groups 1-4), further modified to scparate lakes with profundal sediments rich in carbonate and those rich in organic matter.

(Mortimer 1941, 1942). Depth of penetration ranged from $10-15 \mathrm{~cm}$ in clayey sediments with little organic matter to more than $30 \mathrm{~cm}$ in flocculent highly organic sediments. The top $10 \mathrm{~cm}$ of core used does not represent the same time interval in all cores. Samples wore stored in polyethylenc containers precleaned with hydrochloric acid and rinsed with distilled water.

In the laboratory, samples were air-dried at $90^{\circ} \mathrm{C}$ and ground to powder. Percent organic matter and carbonate were determined by loss on ignition at $550^{\circ} \mathrm{C}$ and $1,000^{\circ} \mathrm{C}$ (Dean 1974). Ignition loss at $550^{\circ} \mathrm{C}$ was assumed to represent ignition of organic matter: this was checked chromatographically with a Hewlett-Packard CHN analyzer (Fig. 3). Ignition loss between $550^{\circ}$ and $1,000^{\circ} \mathrm{C}$ was assumed to represent loss of $\mathrm{CO}_{2}$ from carbonate minerals and percent calcium carbonate calculated by multiplying the percent ignition loss between $550^{\circ}$ and $1,000^{\circ} \mathrm{C}$ by 2.27 , the factor for converting $\mathrm{CO}_{2}$ to $\mathrm{CaCO}_{3}$.

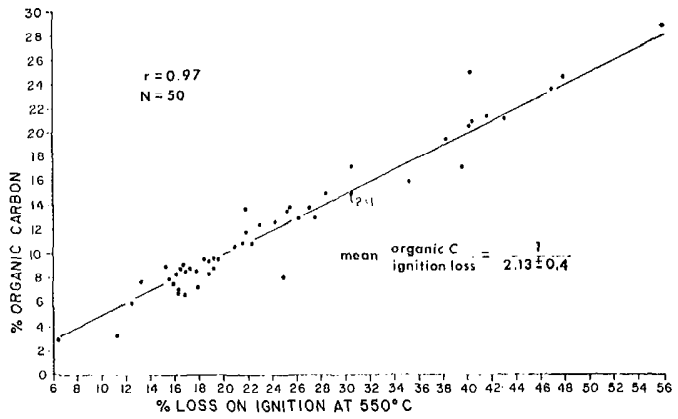

Fig. 3. Scatter plot of organic matter (by loss on ignition at $550^{\circ} \mathrm{C}$ ) and organic carbon in profundal sediments of all 46 lakes.

The accuracy of this method has been checked by comparison with several standard methods (Dean 1974). Lake sediments with considerable clay but no carbonate usually have a 1.0 to $1.8 \%$ weight loss between $550^{\circ}$ and $1,000^{\circ} \mathrm{C}$, presumably due to loss of water from clay lattices; this converts to 2.3 to $4.1 \% \mathrm{CaCO}_{3}$ if we assume that all of the loss is duc to $\mathrm{CO}_{2}$ evolved from carbonate. However, standard additions of $\mathrm{CaCO}_{3}$ to noncalcareous lake sediment show nearly $100 \%$ recovery by the ignition loss method for $\mathrm{CaCO}_{3}$ concentrations above 10\% (Dean 1974). The difference between $100 \%$ and the sum of ignition loss organic mattcr and carbonate was taken as the inorganic, noncarbonate clastic fraction. Ignition loss determinations for organic, carbonate, and clastic components arc given in Table 1 as percent dry weight.

Total and soluble $\mathrm{Ca}$ and $\mathrm{Mg}$ werc determined with an atomic absorption attachment for a Beckman DU spcctrophotometer. Samples ( $500 \mathrm{mg}$ ) for analysis of total $\mathrm{Ca}$ and $\mathrm{Mg}$ were digested and evaporated to dryness successively in $\mathrm{HNO}_{3}, \mathrm{HF}$, and HCl. The final dried residue was taken up in $1.0 \mathrm{~N} \mathrm{HCl}$. For samples high in $\mathrm{Ca}$ and $\mathrm{Mg}, 100$-fold dilutions were necessary. The final solutions contained $1 \%$ lanthanum to suppress interference by silica and alumina. Samples for soluble $\mathrm{Ca}$ and $\mathrm{Mg}$ werc leached with $1.0 \mathrm{~N}$ ammonium acetate at pH 5.2: according to Wangersky and Joensuu (1967) and confirmed here, this 
Table 1. Location, morphometry, and composition of profundal sediments from all 46 lakes. Lakes listed in order of increasing total cation concentration in surface waters.

\begin{tabular}{|c|c|c|c|c|c|c|c|c|c|c|c|c|}
\hline & & & 1 & 2 & 3 & 4 & 5 & 6 & 7 & 8 & 9 & 10 \\
\hline $\begin{array}{l}\text { LAKE } \\
\text { NO. }\end{array}$ & $\begin{array}{l}\text { LAKE } \\
\text { NAME }\end{array}$ & COUNTY & $\begin{array}{l}\text { AREA } \\
\text { SO.KM.) }\end{array}$ & $\begin{array}{l}\text { MAX. } \\
\text { DEPTH } \\
\text { (M) }\end{array}$ & $\begin{array}{c}L .0 .1 . \\
550-C \\
(\%)\end{array}$ & $\begin{array}{c}\text { TOTAL } \\
\text { CARBON } \\
(\%)\end{array}$ & $\begin{array}{c}\text { DRGANIC } \\
\text { CARBON } \\
(\%)\end{array}$ & $\stackrel{H}{H}$ & $\begin{array}{l}N \\
(\mathscr{n})\end{array}$ & $\begin{array}{c}\mathrm{L} .0 . \mathrm{I} \\
1000-\mathrm{C} \\
(\%)\end{array}$ & $\begin{array}{l}\text { TOTAL } \\
\text { CA } \\
(\%)\end{array}$ & $\begin{array}{c}\text { SOLUBLE } \\
\text { CA } \\
(\%)\end{array}$ \\
\hline $\begin{array}{l}1 \\
2 \\
3 \\
4 \\
5 \\
6 \\
7 \\
8 \\
9 \\
10 \\
11 \\
12 \\
13 \\
14 \\
15 \\
16 \\
17 \\
18 \\
19 \\
20 \\
21 \\
22 \\
23 \\
24 \\
25 \\
26 \\
27 \\
28 \\
29 \\
30 \\
31 \\
32 \\
33 \\
34 \\
35 \\
36 \\
37 \\
38 \\
39 \\
40 \\
41 \\
42 \\
43 \\
44 \\
45 \\
46 \\
\end{array}$ & 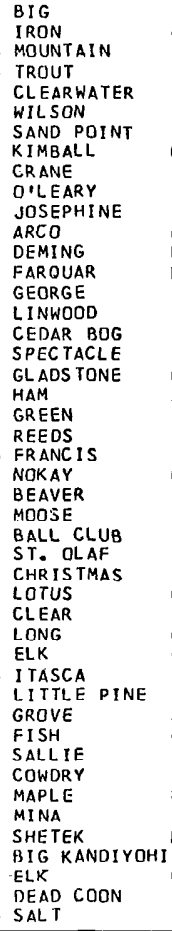 & $\begin{array}{l}\text { ST. LOUIS } \\
\text { COOK } \\
\text { COOK } \\
\text { COOK } \\
\text { COOK } \\
\text { LAKE } \\
\text { ST. LOUIS } \\
\text { COOK } \\
\text { ST. LOUIS } \\
\text { ST: LOUIS } \\
\text { HUBBARD } \\
\text { HUBBARD } \\
\text { HUBBARD } \\
\text { DAKOTA } \\
\text { ANOKA } \\
\text { ANOKA } \\
\text { ANOKA } \\
\text { ISANTI } \\
\text { CROW WING } \\
\text { ANOKA } \\
\text { ISANTI } \\
\text { WASECA } \\
\text { LESUEUR } \\
\text { CROW WING } \\
\text { STEELE } \\
\text { ITASCA } \\
\text { ITASCA } \\
\text { HASECA } \\
\text { HENNEPIN } \\
\text { CARVER } \\
\text { LESUEUR } \\
\text { CLEARWATER } \\
\text { CLEARWATER } \\
\text { CLEARWATER } \\
\text { OTTERTAIL } \\
\text { POPE } \\
\text { COTTONWOOD } \\
\text { BECKER } \\
\text { DOUGLAS } \\
\text { DOUGLAS } \\
\text { DOUGLAS } \\
\text { MURRAY } \\
\text { IKANDIYOHI } \\
\text { GRANT } \\
\text { LINCGLN } \\
\text { LACOUI PARLE }\end{array}$ & $\begin{array}{r}6.88 \\
0.42 \\
8.45 \\
1.05 \\
5.36 \\
2.47 \\
7.33 \\
0.32 \\
12.50 \\
0.80 \\
0.01 \\
0.01 \\
0.01 \\
0.27 \\
2.19 \\
1.98 \\
0.00 \\
0.97 \\
1.95 \\
0.53 \\
3.25 \\
0.76 \\
3.63 \\
2.64 \\
0.40 \\
4.86 \\
15.93 \\
0.41 \\
1.05 \\
0.98 \\
1.27 \\
0.68 \\
1.21 \\
4.36 \\
8.24 \\
1.53 \\
1.21 \\
5.11 \\
0.96 \\
3.38 \\
1.77 \\
17.40 \\
15.38 \\
0.77 \\
2.35 \\
1.00 \\
\end{array}$ & $\begin{array}{r}4.57 \\
5.79 \\
64.01 \\
21.34 \\
39.62 \\
14.02 \\
53.34 \\
5.49 \\
24.38 \\
17.07 \\
11.55 \\
7.60 \\
17.00 \\
1.83 \\
9.75 \\
10.67 \\
1.22 \\
14.33 \\
9.45 \\
6.71 \\
8.53 \\
17.68 \\
17.68 \\
13.11 \\
7.32 \\
21.34 \\
25.91 \\
9.14 \\
27.43 \\
10.67 \\
6.10 \\
24.38 \\
29.57 \\
13.72 \\
19.20 \\
9.45 \\
7.92 \\
14.33 \\
15.85 \\
21.95 \\
37.49 \\
5.49 \\
3.05 \\
7.92 \\
3.35 \\
0.30\end{array}$ & $\begin{array}{r}38.10 \\
40.20 \\
19.20 \\
21.00 \\
15.80 \\
17.40 \\
17.90 \\
46.90 \\
16.90 \\
19.20 \\
40.20 \\
41.60 \\
47.90 \\
16.30 \\
39.50 \\
35.20 \\
60.00 \\
43.00 \\
43.40 \\
27.20 \\
20.90 \\
24.30 \\
26.20 \\
38.40 \\
16.60 \\
6.80 \\
30.60 \\
16.10 \\
18.80 \\
16.40 \\
16.50 \\
12.50 \\
24.20 \\
22.30 \\
17.80 \\
21.90 \\
16.00 \\
18.80 \\
21.50 \\
18.40 \\
17.10 \\
15.40 \\
13.30 \\
15.60 \\
6.40 \\
11.30 \\
\end{array}$ & $\begin{array}{r}18.04 \\
25.31 \\
10.03 \\
9.00 \\
6.11 \\
8.00 \\
7.59 \\
23.94 \\
7.06 \\
9.04 \\
20.92 \\
21.65 \\
25.05 \\
6.98 \\
17.67 \\
17.16 \\
28.98 \\
21.47 \\
21.17 \\
17.19 \\
14.27 \\
13.71 \\
14.06 \\
21.41 \\
12.16 \\
11.81 \\
17.83 \\
10.44 \\
9.53 \\
9.06 \\
10.28 \\
10.82 \\
11.78 \\
15.00 \\
14.14 \\
15.53 \\
9.95 \\
15.05 \\
14.58 \\
14.66 \\
13.48 \\
12.04 \\
10.34 \\
10.13 \\
5.20 \\
5.38\end{array}$ & $\begin{array}{r}17.60 \\
24.90 \\
9.60 \\
8.50 \\
5.80 \\
7.70 \\
7.10 \\
23.50 \\
6.60 \\
8.70 \\
20.60 \\
21.30 \\
24.60 \\
6.70 \\
17.30 \\
15.90 \\
28.60 \\
21.10 \\
20.90 \\
12.70 \\
10.60 \\
17.50 \\
12.80 \\
19.50 \\
9.00 \\
8.40 \\
17.10 \\
8.20 \\
8.30 \\
7.00 \\
8.80 \\
6.00 \\
9.50 \\
10.80 \\
8.50 \\
11.20 \\
7.50 \\
9.50 \\
10.80 \\
9.60 \\
8.80 \\
8.90 \\
7.60 \\
7.80 \\
2.90 \\
3.30\end{array}$ & $\begin{array}{l}2.75 \\
3.55 \\
1.69 \\
1.40 \\
1.03 \\
1.70 \\
1.73 \\
3.58 \\
1.22 \\
1.38 \\
2.89 \\
2.85 \\
3.31 \\
1.05 \\
3.47 \\
3.24 \\
3.95 \\
3.11 \\
3.12 \\
1.78 \\
1.36 \\
1.83 \\
1.87 \\
2.76 \\
1.27 \\
1.19 \\
2.50 \\
1.22 \\
1.25 \\
0.58 \\
1.48 \\
0.82 \\
1.80 \\
1.52 \\
1.17 \\
1.43 \\
1.23 \\
1.51 \\
1.59 \\
1.22 \\
1.49 \\
1.26 \\
1.13 \\
1.35 \\
0.51 \\
0.89\end{array}$ & $\begin{array}{l}1.78 \\
1.79 \\
0.86 \\
1.00 \\
0.50 \\
0.00 \\
0.63 \\
2.85 \\
0.82 \\
0.97 \\
2.09 \\
2.36 \\
2.74 \\
0.92 \\
2.16 \\
1.97 \\
3.47 \\
2.66 \\
2.61 \\
1.39 \\
1.26 \\
1.45 \\
1.60 \\
2.48 \\
0.99 \\
0.90 \\
1.85 \\
0.96 \\
0.99 \\
0.87 \\
0.97 \\
0.64 \\
0.99 \\
1.26 \\
1.11 \\
1.25 \\
0.92 \\
1.20 \\
1.35 \\
1.13 \\
1.04 \\
0.91 \\
0.72 \\
0.81 \\
0.34 \\
0.41\end{array}$ & $\begin{array}{r}3.10 \\
3.70 \\
3.30 \\
4.50 \\
2.80 \\
2.40 \\
4.20 \\
3.60 \\
4.00 \\
2.20 \\
2.50 \\
3.60 \\
3.80 \\
2.60 \\
3.10 \\
10.40 \\
4.20 \\
3.50 \\
3.80 \\
37.30 \\
30.90 \\
10.30 \\
11.10 \\
15.70 \\
26.60 \\
28.30 \\
5.60 \\
18.00 \\
10.30 \\
17.10 \\
12.10 \\
40.30 \\
19.10 \\
34.60 \\
46.30 \\
35.60 \\
21.00 \\
46.40 \\
31.30 \\
42.60 \\
39.40 \\
22.50 \\
22.50 \\
19.00 \\
19.10 \\
17.10\end{array}$ & $\begin{array}{r}0.93 \\
0.84 \\
0.81 \\
1.03 \\
0.84 \\
0.79 \\
0.78 \\
0.88 \\
0.96 \\
1.21 \\
0.87 \\
0.91 \\
0.63 \\
0.80 \\
1.15 \\
5.80 \\
1.14 \\
1.11 \\
1.61 \\
16.40 \\
12.70 \\
3.44 \\
3.51 \\
7.60 \\
10.30 \\
11.00 \\
1.94 \\
6.80 \\
3.61 \\
6.90 \\
4.80 \\
15.20 \\
6.10 \\
14.30 \\
19.90 \\
14.10 \\
7.10 \\
16.50 \\
12.00 \\
15.90 \\
15.30 \\
9.00 \\
9.30 \\
6.80 \\
7.50 \\
6.00\end{array}$ & $\begin{array}{r}0.70 \\
0.70 \\
0.50 \\
0.50 \\
0.50 \\
0.40 \\
0.60 \\
0.50 \\
0.70 \\
0.60 \\
0.90 \\
1.00 \\
0.80 \\
0.60 \\
1.00 \\
5.10 \\
0.90 \\
1.10 \\
1.50 \\
16.50 \\
12.90 \\
1.50 \\
2.50 \\
6.80 \\
9.70 \\
10.70 \\
1.70 \\
6.90 \\
1.50 \\
6.60 \\
4.20 \\
16.20 \\
7.80 \\
12.60 \\
18.10 \\
14.50 \\
6.10 \\
17.10 \\
12.70 \\
16.30 \\
17.70 \\
8.10 \\
8.30 \\
5.30 \\
6.40 \\
5.70\end{array}$ \\
\hline & & $\begin{array}{l}\text { mean } \\
\text { standard dev. }\end{array}$ & $\begin{array}{l}3.20 \\
4.36\end{array}$ & $\begin{array}{l}15.82 \\
12.92\end{array}$ & $\begin{array}{l}24.59 \\
12.24\end{array}$ & $\begin{array}{r}14.02 \\
5.79\end{array}$ & $\begin{array}{r}12.06 \\
6.28\end{array}$ & $\begin{array}{l}1.85 \\
0.90\end{array}$ & $\begin{array}{l}1.36 \\
0.73\end{array}$ & $\begin{array}{l}16.33 \\
14.02\end{array}$ & $\begin{array}{l}6.24 \\
5.69\end{array}$ & $\begin{array}{l}5.93 \\
5.86\end{array}$ \\
\hline & $\begin{array}{l}\text { low organic } \\
\text { Group } 1\end{array}$ & $\begin{array}{l}\text { mean } \\
\text { standard dev. }\end{array}$ & $\begin{array}{l}4.78 \\
4.39\end{array}$ & $\begin{array}{l}29.45 \\
21.09\end{array}$ & $\begin{array}{r}17.96 \\
1.74\end{array}$ & $\begin{array}{l}7.98 \\
1.30\end{array}$ & $\begin{array}{l}7.59 \\
1.27\end{array}$ & $\begin{array}{l}1.40 \\
0.29\end{array}$ & $\begin{array}{l}0.79 \\
0.19\end{array}$ & $\begin{array}{l}3.25 \\
0.88\end{array}$ & $\begin{array}{l}0.90 \\
0.15\end{array}$ & $\begin{array}{l}0.55 \\
0.09\end{array}$ \\
\hline & $\begin{array}{l}\text { high organic } \\
\text { Group } 1 \text { and } 2\end{array}$ & $\begin{array}{l}\text { mean } \\
\text { standard dev. }\end{array}$ & $\begin{array}{l}2.56 \\
4.44\end{array}$ & $\begin{array}{r}11.23 \\
6.38\end{array}$ & $\begin{array}{r}41.92 \\
7.09\end{array}$ & $\begin{array}{r}21.58 \\
3.52\end{array}$ & $\begin{array}{r}20.99 \\
3.66\end{array}$ & $\begin{array}{l}3.16 \\
0.41\end{array}$ & $\begin{array}{l}2.37 \\
0.50\end{array}$ & $\begin{array}{l}5.12 \\
3.75\end{array}$ & $\begin{array}{l}1.95 \\
2.17\end{array}$ & $\begin{array}{l}1.75 \\
1.92\end{array}$ \\
\hline & $\begin{array}{l}\text { intermediate } \\
\text { Group } 2\end{array}$ & $\begin{array}{l}\text { mean } \\
\text { standard dev. }\end{array}$ & $\begin{array}{l}1.58 \\
1.47\end{array}$ & $\begin{array}{r}15.49 \\
8.57\end{array}$ & $\begin{array}{r}18.19 \\
5.63\end{array}$ & $\begin{array}{r}11.28 \\
1.71\end{array}$ & $\begin{array}{l}9.20 \\
1.95\end{array}$ & $\begin{array}{l}1.37 \\
0.39\end{array}$ & $\begin{array}{l}1.06 \\
0.25\end{array}$ & $\begin{array}{r}17.39 \\
6.57\end{array}$ & $\begin{array}{l}6.35 \\
2.68\end{array}$ & $\begin{array}{l}5.75 \\
3.26\end{array}$ \\
\hline & $\begin{array}{l}\text { high carb. } \\
\text { Group } 2\end{array}$ & $\begin{array}{l}\text { mean } \\
\text { standard dev. }\end{array}$ & $\begin{array}{l}2.98 \\
2.44\end{array}$ & $\begin{array}{r}17.16 \\
9.17\end{array}$ & $\begin{array}{r}19.84 \\
3.90\end{array}$ & $\begin{array}{r}14.47 \\
1.62\end{array}$ & $\begin{array}{l}9.85 \\
1.83\end{array}$ & $\begin{array}{l}1.39 \\
0.27\end{array}$ & $\begin{array}{l}1.16 \\
0.21\end{array}$ & $\begin{array}{r}38.47 \\
5.56\end{array}$ & $\begin{array}{r}15.23 \\
2.21\end{array}$ & $\begin{array}{r}15.41 \\
2.10\end{array}$ \\
\hline & Group 3 & $\begin{array}{l}\text { mean } \\
\text { standard dev. }\end{array}$ & $\begin{array}{l}7.38 \\
8.28\end{array}$ & $\begin{array}{l}4.02 \\
2.85\end{array}$ & $\begin{array}{r}12.40 \\
3.78\end{array}$ & $\begin{array}{l}8.62 \\
3.12\end{array}$ & $\begin{array}{l}6.10 \\
2.79\end{array}$ & $\begin{array}{l}1.23 \\
0.314\end{array}$ & $\begin{array}{l}0.64 \\
0.25\end{array}$ & $\begin{array}{r}20.05 \\
2.38 \\
\end{array}$ & $\begin{array}{l}7.72 \\
1.41\end{array}$ & $\begin{array}{l}6.76 \\
1.37 \\
\end{array}$ \\
\hline
\end{tabular}

reagent dissolves calcite and aragonite but not dolomite. Results are given in Table 1 as percent dry weight of sample.

Sedimonts for size analyses were pretrcated with $1.0 \mathrm{~N}$ ammonium acetate at $\mathrm{pHI} 5.2$ to remove calcium carbonate and $30 \% \mathrm{H}_{2} \mathrm{O}_{2}$ to remove most of the organic fraction. They were then washed through a $44-\mu$ nylon sieve. The sand-size fraction retained on the sieve was washed, dried, weighed, and mounted on slides for mineralogical analyses. Material passing the sieve (silt + clay) was centrifuged to separate silt and clay fractions (Jackson 1967). After centrifugation, the supernatant containing the clay was filtered through a $0.45-\mu$ Millipore filter and saved for X-ray analysis. The silt-size fraction remaining 
Table 1. Continued.

\begin{tabular}{|c|c|c|c|c|c|c|c|c|c|c|c|c|}
\hline & & & & 11 & 12 & 13 & 14 & 15 & 16 & 17 & 18 & 19 \\
\hline & $\begin{array}{l}\text { AKE } \\
\text { NO. }\end{array}$ & $\begin{array}{l}\text { LAKE } \\
\text { NAME }\end{array}$ & COUNTY & $\begin{array}{l}X-R A Y \\
\text { CALCITE } \\
\text { (CM) }\end{array}$ & $\begin{array}{l}\text { X-RAY } \\
\text { ARAGON. } \\
\text { (CM) }\end{array}$ & $\begin{array}{l}X-R A Y \\
\text { DOLOM. } \\
\text { (CM) }\end{array}$ & $\begin{array}{l}\text { TOTAL } \\
M G \\
(\&)\end{array}$ & $\begin{array}{c}\text { SOLURLE } \\
M G \\
(\%)\end{array}$ & $\begin{array}{l}\text { CLASTIC } \\
(\%)\end{array}$ & $\begin{array}{l}\text { SANO } \\
\text { (\%) }\end{array}$ & $\begin{array}{l}\text { SILT } \\
\left.(\Psi)^{\prime}\right)\end{array}$ & $\begin{array}{r}\text { CLAY } \\
(\%)\end{array}$ \\
\hline $\begin{array}{l}\text { N } \\
0 \\
a \\
0 \\
0 \\
\simeq \\
0\end{array}$ & $\begin{array}{l}1 \\
2 \\
3 \\
4 \\
5 \\
6 \\
7 \\
8 \\
9 \\
10 \\
11 \\
12 \\
13 \\
14 \\
15 \\
16 \\
17 \\
18 \\
19 \\
20 \\
21 \\
22 \\
23 \\
24 \\
25 \\
26 \\
27 \\
28 \\
29 \\
30 \\
31 \\
32 \\
33 \\
34 \\
35 \\
36 \\
37 \\
38 \\
39 \\
40 \\
41 \\
42 \\
43 \\
44 \\
45 \\
46\end{array}$ & 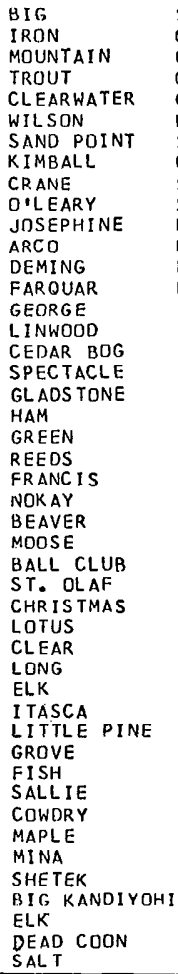 & $\begin{array}{l}\text { ST. LOUIS } \\
\text { COOK } \\
\text { COOK } \\
\text { COOK } \\
\text { COOK } \\
\text { LAKE } \\
\text { ST. LOUIS } \\
\text { COOK } \\
\text { ST. LOUIS } \\
\text { ST. LOUIS } \\
\text { HUBBARD } \\
\text { IIUBBARD } \\
\text { RUBBARD } \\
\text { DAKOTA } \\
\text { ANOKA } \\
\text { ANOKA } \\
\text { ANOKA } \\
\text { ISANTI } \\
\text { CROW WING } \\
\text { ANOKA } \\
\text { ISANTI } \\
\text { WASECA } \\
\text { LESUEUR } \\
\text { CROW WING } \\
\text { STEELE } \\
\text { ITASCA } \\
\text { ITASCA } \\
\text { WASECA } \\
\text { HENNEPIN } \\
\text { CARVER } \\
\text { LESUEUR } \\
\text { CLEARWATER } \\
\text { CLEARWATER } \\
\text { CLEARWATER } \\
\text { OTTERTAIL } \\
\text { POPE } \\
\text { COTTDNWOOD } \\
\text { BECKER } \\
\text { DOUGLAS } \\
\text { DOUGLAS } \\
\text { OOUGLAS } \\
\text { MURRAY } \\
\text { IKANOIYOHI } \\
\text { GRANT } \\
\text { LINCOLN } \\
\text { LACOUI PARLE }\end{array}$ & $\begin{array}{c}0.0 \\
0.0 \\
0.0 \\
0.0 \\
0.0 \\
0.0 \\
0.0 \\
0.0 \\
0.0 \\
0.0 \\
0.0 \\
0.0 \\
0.0 \\
0.0 \\
0.0 \\
10.16 \\
0.0 \\
0.0 \\
0.0 \\
25.15 \\
20.32 \\
4.32 \\
2.54 \\
8.89 \\
13.97 \\
16.51 \\
1.27 \\
11.43 \\
5.08 \\
4.57 \\
5.08 \\
25.15 \\
0.0 \\
25.15 \\
25.15 \\
21.59 \\
11.43 \\
25.15 \\
19.05 \\
16.51 \\
22.86 \\
17.78 \\
16.51 \\
25.15 \\
7.62 \\
6.35\end{array}$ & $\begin{array}{l}0.0 \\
0.0 \\
0.0 \\
0.0 \\
0.0 \\
0.0 \\
0.0 \\
0.0 \\
0.0 \\
0.0 \\
0.0 \\
0.0 \\
0.0 \\
0.0 \\
0.0 \\
0.0 \\
0.0 \\
0.0 \\
0.0 \\
0.0 \\
0.0 \\
0.0 \\
0.0 \\
0.0 \\
0.0 \\
0.0 \\
0.0 \\
0.0 \\
0.0 \\
0.0 \\
0.0 \\
0.0 \\
0.0 \\
0.51 \\
0.0 \\
0.0 \\
1.02 \\
0.0 \\
1.02 \\
1.27 \\
0.76 \\
0.0 \\
1.02 \\
1.02 \\
0.0 \\
0.0 \\
\end{array}$ & $\begin{array}{l}0.0 \\
0.0 \\
0.0 \\
0.0 \\
0.0 \\
0.0 \\
0.0 \\
0.0 \\
0.0 \\
0.0 \\
0.0 \\
0.0 \\
0.0 \\
0.0 \\
0.0 \\
2.79 \\
0.0 \\
0.0 \\
0.0 \\
0.0 \\
0.76 \\
2.03 \\
3.56 \\
0.0 \\
1.78 \\
2.29 \\
0.0 \\
1.78 \\
2.54 \\
1.02 \\
1.02 \\
1.02 \\
0.0 \\
0.0 \\
0.51 \\
0.0 \\
1.02 \\
1.02 \\
1.02 \\
1.02 \\
0.0 \\
1.78 \\
2.29 \\
5.59 \\
4.32 \\
1.52\end{array}$ & $\begin{array}{l}0.29 \\
0.44 \\
0.74 \\
0.44 \\
0.88 \\
0.82 \\
0.56 \\
0.53 \\
0.87 \\
0.93 \\
0.51 \\
0.51 \\
0.43 \\
0.62 \\
0.52 \\
0.32 \\
0.24 \\
0.44 \\
0.45 \\
0.48 \\
0.48 \\
0.98 \\
1.20 \\
0.46 \\
1.13 \\
1.45 \\
0.55 \\
0.98 \\
0.84 \\
1.00 \\
1.09 \\
1.08 \\
0.45 \\
0.57 \\
0.71 \\
0.93 \\
1.11 \\
1.23 \\
0.94 \\
1.16 \\
0.90 \\
1.66 \\
1.14 \\
2.79 \\
1.28 \\
1.46\end{array}$ & $\begin{array}{l}0.10 \\
0.07 \\
0.05 \\
0.12 \\
0.13 \\
0.10 \\
0.04 \\
0.08 \\
0.07 \\
0.08 \\
0.08 \\
0.10 \\
0.08 \\
0.08 \\
0.13 \\
0.15 \\
0.08 \\
0.13 \\
0.15 \\
0.25 \\
0.23 \\
0.38 \\
0.64 \\
0.16 \\
0.44 \\
0.77 \\
0.28 \\
0.42 \\
0.46 \\
0.46 \\
0.53 \\
0.64 \\
0.30 \\
0.34 \\
0.45 \\
0.52 \\
0.58 \\
0.72 \\
0.67 \\
0.79 \\
0.68 \\
0.93 \\
0.39 \\
0.78 \\
0.44 \\
0.59\end{array}$ & $\begin{array}{l}58.80 \\
56.10 \\
77.50 \\
74.50 \\
81.40 \\
80.20 \\
77.90 \\
49.50 \\
79.10 \\
78.60 \\
57.30 \\
54.80 \\
48.30 \\
81.10 \\
57.40 \\
54.40 \\
35.80 \\
53.50 \\
52.80 \\
35.50 \\
48.20 \\
65.40 \\
62.70 \\
45.90 \\
56.80 \\
54.90 \\
63.80 \\
65.90 \\
70.90 \\
66.50 \\
71.40 \\
47.20 \\
56.70 \\
43.10 \\
35.90 \\
42.50 \\
63.000 \\
34.80 \\
47.20 \\
39.00 \\
43.50 \\
62.10 \\
64.20 \\
65.40 \\
74.50 \\
71.40\end{array}$ & $\begin{array}{r}0.70 \\
1.30 \\
0.60 \\
0.30 \\
2.60 \\
23.50 \\
13.80 \\
0.30 \\
0.10 \\
2.60 \\
1.50 \\
0.60 \\
0.70 \\
0.40 \\
2.10 \\
1.20 \\
0.30 \\
2.20 \\
6.90 \\
2.20 \\
1.80 \\
1.50 \\
2.10 \\
2.00 \\
6.60 \\
5.80 \\
11.50 \\
1.40 \\
1.00 \\
1.30 \\
0.70 \\
2.30 \\
0.30 \\
0.20 \\
1.50 \\
0.30 \\
2.40 \\
0.90 \\
0.30 \\
1.550 \\
5.90 \\
2.10 \\
1.60 \\
6.10 \\
5.40 \\
1.90\end{array}$ & $\begin{array}{r}19.60 \\
18.70 \\
31.20 \\
30.10 \\
33.80 \\
21.70 \\
46.60 \\
34.90 \\
22.80 \\
32.30 \\
23.50 \\
25.60 \\
18.20 \\
15.40 \\
25.50 \\
21.40 \\
10.40 \\
23.90 \\
29.90 \\
9.90 \\
15.70 \\
25.10 \\
24.70 \\
25.00 \\
30.00 \\
17.50 \\
28.70 \\
34.30 \\
31.70 \\
27.10 \\
16.70 \\
24.20 \\
18.00 \\
9.40 \\
11.60 \\
16.70 \\
26.70 \\
9.90 \\
18.70 \\
11.40 \\
19.50 \\
22.90 \\
36.40 \\
21.30 \\
2.9 .10 \\
21.30\end{array}$ & $\begin{array}{r}38.00 \\
21.40 \\
31.80 \\
37.00 \\
32.80 \\
34.40 \\
16.40 \\
22.20 \\
43.20 \\
24.40 \\
21.80 \\
18.40 \\
16.00 \\
24.60 \\
25.20 \\
19.00 \\
12.80 \\
16.60 \\
15.20 \\
12.80 \\
15.20 \\
24.60 \\
24.40 \\
25.00 \\
25.40 \\
24.20 \\
16.80 \\
24.60 \\
24.00 \\
40.40 \\
32.40 \\
11.20 \\
7.20 \\
9.80 \\
13.20 \\
18.00 \\
27.60 \\
14.600 \\
16.40 \\
12.40 \\
15.40 \\
17.60 \\
22.20 \\
21.00 \\
29.00 \\
29.00\end{array}$ \\
\hline & & & $\begin{array}{l}\text { mean } \\
\text { standard dev. }\end{array}$ & $\begin{array}{l}8.59 \\
9.58\end{array}$ & $\begin{array}{l}0.15 \\
0.40\end{array}$ & $\begin{array}{l}0.88 \\
1.29\end{array}$ & $\begin{array}{l}0.84 \\
0.45\end{array}$ & $\begin{array}{l}0.34 \\
0.26\end{array}$ & $\begin{array}{l}58.86 \\
13.69\end{array}$ & $\begin{array}{l}2.88 \\
4.22\end{array}$ & $\begin{array}{r}23.39 \\
8.08\end{array}$ & $\begin{array}{r}22.30 \\
8.39\end{array}$ \\
\hline & & $\begin{array}{l}\text { ow organic } \\
\text { roup } 1\end{array}$ & $\begin{array}{l}\text { mean } \\
\text { standard dev. }\end{array}$ & $\begin{array}{l}0.00 \\
0.00\end{array}$ & $\begin{array}{l}0.00 \\
0.00\end{array}$ & $\begin{array}{l}0.00 \\
0.00\end{array}$ & $\begin{array}{l}0.73 \\
0.18\end{array}$ & $\begin{array}{l}0.08 \\
0.03\end{array}$ & $\begin{array}{r}78.78 \\
2.24\end{array}$ & $\begin{array}{l}5.49 \\
8.59\end{array}$ & $\begin{array}{r}29.24 \\
9.46\end{array}$ & $\begin{array}{r}30.58 \\
8.42\end{array}$ \\
\hline & & $\begin{array}{l}\text { igh organic } \\
\text { roup } 1 \text { and } 2\end{array}$ & $\begin{array}{l}\text { mean } \\
\text { standard dev. }\end{array}$ & $\begin{array}{l}1.56 \\
3.56\end{array}$ & $\begin{array}{l}0.00 \\
0.00\end{array}$ & $\begin{array}{l}0.21 \\
0.77\end{array}$ & $\begin{array}{l}0.43 \\
0.10\end{array}$ & $\begin{array}{l}0.12 \\
0.06\end{array}$ & $\begin{array}{r}52.95 \\
6.97\end{array}$ & $\begin{array}{l}2.40 \\
3.22\end{array}$ & $\begin{array}{r}23.48 \\
6.13\end{array}$ & $\begin{array}{r}20.65 \\
6.43\end{array}$ \\
\hline & & $\begin{array}{l}\text { ntermediate } \\
\text { roup } 2\end{array}$ & $\begin{array}{l}\text { mean } \\
\text { standard dev. }\end{array}$ & $\begin{array}{l}7.49 \\
5.43\end{array}$ & $\begin{array}{l}0.10 \\
0.32\end{array}$ & $\begin{array}{l}1.70 \\
1.00\end{array}$ & $\begin{array}{l}1.02 \\
0.26\end{array}$ & $\begin{array}{l}0.50 \\
0.14\end{array}$ & $\begin{array}{r}63.42 \\
5.79\end{array}$ & $\begin{array}{l}2.39 \\
2.10\end{array}$ & $\begin{array}{r}25.18 \\
6.12\end{array}$ & $\begin{array}{r}25.48 \\
8.27\end{array}$ \\
\hline & & $\begin{array}{l}\text { igh carb. } \\
\text { roup } 2\end{array}$ & $\begin{array}{l}\text { mean } \\
\text { standard dev. }\end{array}$ & $\begin{array}{r}22.61 \\
3.14\end{array}$ & $\begin{array}{l}0.38 \\
0.54\end{array}$ & $\begin{array}{l}0.54 \\
0.49\end{array}$ & $\begin{array}{l}0.85 \\
0.28\end{array}$ & $\begin{array}{l}0.53 \\
0.20\end{array}$ & $\begin{array}{r}41.69 \\
5.12\end{array}$ & $\begin{array}{l}1.69 \\
1.67\end{array}$ & $\begin{array}{r}14.70 \\
5.04\end{array}$ & $\begin{array}{r}13.90 \\
2.48\end{array}$ \\
\hline & & roup 3 & $\begin{array}{l}\text { mean } \\
\text { standard dev. }\end{array}$ & $\begin{array}{r}14.68 \\
\quad 7.78 \\
\end{array}$ & $\begin{array}{l}0.51 \\
0.72 \\
\end{array}$ & $\begin{array}{l}3.10 \\
1.77\end{array}$ & $\begin{array}{l}1.67 \\
0.66\end{array}$ & $\begin{array}{l}0.63 \\
0.23\end{array}$ & $\begin{array}{r}67.56 \\
5.25\end{array}$ & $\begin{array}{l}3.42 \\
2.15\end{array}$ & $\begin{array}{r}27.60 \\
5.96\end{array}$ & $\begin{array}{r}23.76 \\
5.07\end{array}$ \\
\hline
\end{tabular}

in the centrifuge tube was dried and mounted on glass slides for X-ray analysis. Results of size analyses are given in Table 1.

Sand grains were mounted on a glass slide with epoxy cement and ground with 400 -mesh carborundum grit to give a flat surface, then etched with HF fumes, and stained with sodium colbaltinitrite and amaranth (Boone and Wheeler 1968). This proccdure stains plagioclase red, orthoclase yellow, and leaves quartz grains clear. Percentages of quartz, plagioclase, orthoclase, and accessory minerals were determined by point-counting at least 100 grains under a petrographic microscope. Only 23 samples contained enough sand grains to be counted (Table 2).

Mineralogy of the silt-size fraction was 
Table 1. Continued.

\begin{tabular}{|c|c|c|c|c|c|c|c|c|c|c|c|}
\hline \multirow{2}{*}{\multicolumn{2}{|c|}{$\begin{array}{l}\text { LAKE } \\
\text { NO. }\end{array}$}} & \multirow[b]{2}{*}{$\begin{array}{l}\text { LAKE } \\
\text { NAME }\end{array}$} & \multirow[b]{2}{*}{ COUNTY } & \multirow{2}{*}{$\begin{array}{r}20 \\
X-R A Y \\
4.26 \AA \\
\text { DUARTZ } \\
\text { (CM) }\end{array}$} & \multirow{2}{*}{$\begin{array}{c}21 \\
\text { PEAK HTS } \\
4.20 \AA \\
\text { F'SPAR } \\
\text { (CM) }\end{array}$} & \multirow{2}{*}{$\begin{array}{c}22 \\
2-44 \mu \\
4.18 \AA \\
F(S P A R \\
\text { (CM) }\end{array}$} & \multirow{2}{*}{$\begin{array}{l}\frac{23}{\text { RELATIVE }} \\
\text { ILLITE } \\
\text { (CLAY) }\end{array}$} & \multirow{2}{*}{$\begin{array}{l}24 \\
\text { E PEAK } \\
\text { CHLOR. } \\
\text { (CLAY) }\end{array}$} & \multirow{2}{*}{$\begin{array}{l}25 \\
\text { HEIGHTS } \\
\text { QUARTZ } \\
\text { (CLAY) }\end{array}$} & \multirow{2}{*}{$\begin{array}{c}26 \\
\text { (GLYCOL) } \\
\text { F'SPAR } \\
\text { (CLAY) }\end{array}$} & \multirow{2}{*}{$\begin{array}{l}27 \\
\text { X-RAY } \\
\text { TOTAL } \\
\text { QUARTZ } \\
\text { (CM) }\end{array}$} \\
\hline & & & & & & & & & & & \\
\hline $\begin{array}{l}- \\
a \\
0 \\
0 \\
x \\
0\end{array}$ & $\begin{array}{l}1 \\
2 \\
3 \\
4 \\
5 \\
6 \\
7 \\
8 \\
9 \\
10 \\
11 \\
12 \\
13 \\
14 \\
15 \\
16 \\
17 \\
18 \\
19 \\
20 \\
21 \\
22 \\
23 \\
24 \\
25 \\
26 \\
27 \\
28 \\
29 \\
30 \\
31 \\
32 \\
33 \\
34 \\
35 \\
36 \\
37 \\
38 \\
39 \\
40 \\
41 \\
42 \\
43 \\
44 \\
45 \\
46 \\
\end{array}$ & $\begin{array}{l}\text { BIG } \\
\text { IRON } \\
\text { MOUNTAIN } \\
\text { TROUT } \\
\text { CLEARWATIR } \\
\text { WILSON } \\
\text { SANO POINT } \\
\text { KIMBALL } \\
\text { CRANE } \\
\text { OLEARY } \\
\text { JOSEPHINE } \\
\text { ARCO } \\
\text { DEMING } \\
\text { FAROUAR } \\
\text { GEORGE } \\
\text { LINWOOD } \\
\text { CEDAR BOG } \\
\text { SPECTACLE } \\
\text { GLADSTONE } \\
\text { HAM } \\
\text { GREEN } \\
\text { REEDS } \\
\text { FRANCIS } \\
\text { NOKAY } \\
\text { BEAVER } \\
\text { MOOSE } \\
\text { BALL CLUB } \\
\text { ST. OLAF } \\
\text { CHRISTMAS } \\
\text { LOTUS } \\
\text { CLEAR } \\
\text { LONG } \\
\text { ELK } \\
\text { ITASCA } \\
\text { LITTLE PINE } \\
\text { GROVE } \\
\text { FISH } \\
\text { SALLIE } \\
\text { COWORY } \\
\text { MAPLE } \\
\text { MINA } \\
\text { SHETEK } \\
\text { BIG KANOIYOH } \\
\text { ELK } \\
\text { DEAD COON } \\
\text { SALT } \\
\end{array}$ & $\begin{array}{l}\text { ST. LQUIS } \\
\text { CDOK } \\
\text { COOK } \\
\text { COOK } \\
\text { COOK } \\
\text { LAKE } \\
\text { ST. LOUIS } \\
\text { COOK } \\
\text { ST. LOUIS } \\
\text { ST. LOUIS } \\
\text { HUBBARD } \\
\text { HUBBARD } \\
\text { HUBBARD } \\
\text { DAKOTA } \\
\text { ANOKA } \\
\text { ANOKA } \\
\text { ANOKA } \\
\text { ISANTI } \\
\text { CROW WING } \\
\text { ANOKA } \\
\text { ISANTI } \\
\text { WASECA } \\
\text { LESUEUR } \\
\text { CROW WING } \\
\text { STEELE } \\
\text { ITASCA } \\
\text { ITASCA } \\
\text { WASECA } \\
\text { HENNEPIN } \\
\text { CARVER } \\
\text { LESUEUR } \\
\text { CLEARWATER } \\
\text { CLEARWATER } \\
\text { CLEARWATER } \\
\text { OTTERTAIL } \\
\text { POPE } \\
\text { COTTDNWOOD } \\
\text { BECKER } \\
\text { DOUGLAS } \\
\text { DOUGLAS } \\
\text { DOUGLAS } \\
\text { MURRAY } \\
\text { IIKANDIYOHI } \\
\text { GRANT } \\
\text { LINCOLN } \\
\text { LACOUI PARLE }\end{array}$ & $\begin{array}{l}1.27 \\
1.52 \\
1.27 \\
1.27 \\
4.57 \\
1.52 \\
1.27 \\
2.54 \\
4.57 \\
3.81 \\
5.08 \\
6.10 \\
5.08 \\
4.57 \\
3.56 \\
1.78 \\
4.83 \\
4.32 \\
0.0 \\
3.05 \\
2.03 \\
3.81 \\
6.35 \\
2.29 \\
5.33 \\
6.60 \\
0.76 \\
6.35 \\
3.56 \\
5.08 \\
7.62 \\
2.54 \\
4.06 \\
3.56 \\
4.83 \\
5.33 \\
6.86 \\
6.35 \\
2.54 \\
6.60 \\
3.30 \\
5.33 \\
5.59 \\
5.59 \\
7.11 \\
7.37\end{array}$ & $\begin{array}{l}1.27 \\
1.02 \\
0.25 \\
0.25 \\
0.0 \\
1.02 \\
1.27 \\
3.05 \\
3.05 \\
1.78 \\
4.57 \\
2.54 \\
2.54 \\
1.78 \\
0.25 \\
1.27 \\
3.56 \\
2.54 \\
0.0 \\
2.54 \\
1.78 \\
0.0 \\
3.56 \\
3.05 \\
0.0 \\
3.81 \\
0.0 \\
0.0 \\
0.0 \\
2.54 \\
4.06 \\
0.51 \\
1.27 \\
5.59 \\
6.60 \\
2.54 \\
2.03 \\
4.57 \\
2.03 \\
4.06 \\
3.30 \\
1.52 \\
2.03 \\
1.52 \\
0.0 \\
2.79\end{array}$ & $\begin{array}{l}2.54 \\
4.00 \\
2.03 \\
1.02 \\
0.0 \\
3.56 \\
2.54 \\
5.84 \\
5.84 \\
4.57 \\
8.64 \\
3.81 \\
5.84 \\
2.54 \\
2.54 \\
2.79 \\
4.83 \\
3.05 \\
0.0 \\
4.57 \\
2.29 \\
0.0 \\
5.33 \\
3.30 \\
2.79 \\
8.38 \\
0.25 \\
0.0 \\
2.54 \\
3.56 \\
5.33 \\
2.29 \\
2.79 \\
6.86 \\
6.35 \\
4.006 \\
3.81 \\
6.86 \\
3.30 \\
5.33 \\
4.06 \\
2.29 \\
2.03 \\
2.54 \\
2.29 \\
4.06\end{array}$ & $\begin{array}{l}1.00 \\
0.0 \\
2.00 \\
0.0 \\
2.00 \\
1.00 \\
0.0 \\
1.00 \\
1.00 \\
2.00 \\
1.00 \\
1.00 \\
1.00 \\
2.00 \\
1.00 \\
0.0 \\
0.0 \\
0.0 \\
0.0 \\
0.0 \\
0.0 \\
2.00 \\
2.00 \\
1.00 \\
4.00 \\
2.00 \\
0.0 \\
2.00 \\
2.00 \\
2.00 \\
2.00 \\
2.00 \\
0.0 \\
0.0 \\
1.00 \\
2.00 \\
2.00 \\
2.00 \\
1.00 \\
2.00 \\
1.00 \\
2.00 \\
2.00 \\
3.00 \\
3.00 \\
2.00\end{array}$ & $\begin{array}{l}1.00 \\
0.0 \\
2.00 \\
1.00 \\
2.00 \\
1.00 \\
1.00 \\
0.0 \\
2.00 \\
2.00 \\
2.00 \\
2.00 \\
2.00 \\
2.00 \\
0.0 \\
0.0 \\
0.0 \\
0.0 \\
0.0 \\
0.0 \\
1.00 \\
2.00 \\
2.00 \\
1.00 \\
2.00 \\
2.00 \\
0.0 \\
2.00 \\
2.00 \\
2.00 \\
2.00 \\
2.00 \\
0.0 \\
0.0 \\
0.0 \\
2.00 \\
2.00 \\
2.00 \\
1.00 \\
2.00 \\
1.00 \\
2.00 \\
3.00 \\
3.00 \\
3.00 \\
2.00\end{array}$ & $\begin{array}{l}3.00 \\
1.00 \\
2.00 \\
1.00 \\
2.00 \\
1.00 \\
1.00 \\
1.00 \\
2.00 \\
2.00 \\
2.00 \\
2.00 \\
2.00 \\
2.00 \\
1.00 \\
1.00 \\
0.0 \\
1.00 \\
1.00 \\
0.0 \\
1.00 \\
2.00 \\
2.00 \\
1.00 \\
2.00 \\
2.00 \\
2.00 \\
2.00 \\
2.00 \\
2.00 \\
2.00 \\
2.00 \\
1.00 \\
1.00 \\
2.00 \\
2.00 \\
2.00 \\
2.00 \\
2.00 \\
2.00 \\
2.00 \\
2.00 \\
2.00 \\
2.00 \\
2.00 \\
2.00\end{array}$ & $\begin{array}{l}2.00 \\
0.0 \\
1.00 \\
0.0 \\
0.0 \\
1.00 \\
1.00 \\
1.00 \\
1.00 \\
2.00 \\
2.00 \\
2.00 \\
2.00 \\
1.00 \\
1.00 \\
0.0 \\
0.0 \\
0.0 \\
1.00 \\
0.0 \\
0.0 \\
1.00 \\
2.00 \\
0.0 \\
1.00 \\
1.00 \\
0.0 \\
2.00 \\
2.00 \\
2.00 \\
2.00 \\
2.00 \\
0.0 \\
0.0 \\
2.00 \\
1.00 \\
0.0 \\
2.00 \\
1.00 \\
2.00 \\
1.00 \\
1.00 \\
1.00 \\
1.00 \\
2.00 \\
2.00\end{array}$ & $\begin{array}{r}3.81 \\
3.35 \\
7.62 \\
7.62 \\
8.89 \\
15.24 \\
5.08 \\
8.89 \\
11.43 \\
25.15 \\
17.78 \\
16.51 \\
17.78 \\
25.15 \\
10.16 \\
6.35 \\
0.0 \\
19.05 \\
0.0 \\
10.16 \\
8.89 \\
17.78 \\
10.16 \\
6.35 \\
13.97 \\
8.89 \\
8.89 \\
13.97 \\
19.05 \\
7.62 \\
16.51 \\
8.89 \\
0.0 \\
3.05 \\
2.54 \\
8.89 \\
13.97 \\
5.08 \\
6.35 \\
5.08 \\
5.08 \\
15.24 \\
15.24 \\
13.97 \\
22.86 \\
16.51\end{array}$ \\
\hline & & & $\begin{array}{l}\text { mean } \\
\text { standard dev. }\end{array}$ & $\begin{array}{l}4.10 \\
2.00\end{array}$ & $\begin{array}{l}2.04 \\
1.63\end{array}$ & $\begin{array}{l}3.55 \\
2.08\end{array}$ & $\begin{array}{l}1.30 \\
0.99\end{array}$ & $\begin{array}{l}1.36 \\
0.96\end{array}$ & $\begin{array}{l}1.65 \\
0.60\end{array}$ & $\begin{array}{l}1.07 \\
0.80\end{array}$ & $\begin{array}{r}10.81 \\
6.38\end{array}$ \\
\hline & $\begin{array}{l}101 \\
\text { Gr }\end{array}$ & $\begin{array}{l}\text { w organic } \\
\text { oup } 1\end{array}$ & $\begin{array}{l}\text { mean } \\
\text { standard dev. }\end{array}$ & $\begin{array}{l}2.86 \\
1.65\end{array}$ & $\begin{array}{l}1.18 \\
1.03\end{array}$ & $\begin{array}{l}2.76 \\
1.88\end{array}$ & $\begin{array}{l}1.25 \\
0.89\end{array}$ & $\begin{array}{l}1.63 \\
0.52\end{array}$ & $\begin{array}{l}1.63 \\
0.52\end{array}$ & $\begin{array}{l}0.88 \\
0.64\end{array}$ & $\begin{array}{r}13.27 \\
7.93\end{array}$ \\
\hline & $\begin{array}{l}\text { hi } \\
\text { Gr }\end{array}$ & $\begin{array}{l}\text { gh organic } \\
\text { oup } 1 \text { and } 2\end{array}$ & $\begin{array}{l}\text { mean } \\
\text { standard dev. }\end{array}$ & $\begin{array}{l}3.01 \\
1.94\end{array}$ & $\begin{array}{l}1.97 \\
1.45\end{array}$ & $\begin{array}{l}3.65 \\
2.32\end{array}$ & $\begin{array}{l}0.54 \\
0.52\end{array}$ & $\begin{array}{l}0.62 \\
0.87\end{array}$ & $\begin{array}{l}1.38 \\
0.77\end{array}$ & $\begin{array}{l}0.85 \\
0.90\end{array}$ & $\begin{array}{l}9.38 \\
6.59\end{array}$ \\
\hline & in & $\begin{array}{l}\text { termediate } \\
\text { oup } 2\end{array}$ & $\begin{array}{l}\text { mean } \\
\text { standard dev. }\end{array}$ & $\begin{array}{l}5.56 \\
1.41\end{array}$ & $\begin{array}{l}1.73 \\
1.70\end{array}$ & $\begin{array}{l}3.45 \\
2.52\end{array}$ & $\begin{array}{l}2.00 \\
0.94\end{array}$ & $\begin{array}{l}1.80 \\
0.63\end{array}$ & $\begin{array}{l}1.90 \\
0.32\end{array}$ & $\begin{array}{l}1.30 \\
0.82\end{array}$ & $\begin{array}{r}12.19 \\
5.69\end{array}$ \\
\hline & $\begin{array}{l}\text { hig } \\
\text { Gre }\end{array}$ & $\begin{array}{l}\text { gh carb. } \\
\text { oup } 2\end{array}$ & $\begin{array}{l}\text { mean } \\
\text { standard dev. }\end{array}$ & $\begin{array}{l}4.01 \\
1.65\end{array}$ & $\begin{array}{l}3.35 \\
1.86\end{array}$ & $\begin{array}{l}4.60 \\
1.72\end{array}$ & $\begin{array}{l}1.10 \\
0.88\end{array}$ & $\begin{array}{l}1.10 \\
0.88\end{array}$ & $\begin{array}{l}1.60 \\
0.70\end{array}$ & $\begin{array}{l}1.10 \\
0.88\end{array}$ & $\begin{array}{l}6.40 \\
2.67\end{array}$ \\
\hline & Grc & oup 3 & $\begin{array}{l}\text { mean } \\
\text { standard dev. }\end{array}$ & $\begin{array}{l}6.20 \\
0.96\end{array}$ & $\begin{array}{l}1.57 \\
1.02\end{array}$ & $\begin{array}{l}2.64 \\
0.81\end{array}$ & $\begin{array}{l}2.40 \\
0.55\end{array}$ & $\begin{array}{l}2.60 \\
0.55\end{array}$ & $\begin{array}{l}2.00 \\
0.00\end{array}$ & $\begin{array}{l}1.40 \\
0.55\end{array}$ & $\begin{array}{r}16.76 \\
3.52\end{array}$ \\
\hline
\end{tabular}

determined by X-ray diffraction with a Gencral Electric XRD-5 X-ray unit with Ni-filtered $\mathrm{Cu}-\mathrm{K} \alpha$ radiation, $35 \mathrm{kV}, 24 \mathrm{~mA}$, $1^{\circ}$ divergence slit, $0.1^{\circ}$ receiving slit, 4-s time constant, full-scale deflection of 4,000 cps, scan rate of $2^{\circ} 2 \theta$ per minute, and chart speed of 1 inch $(2.54 \mathrm{~cm})$ per minute. Represcntative patterns are shown in Fig. 4. Results of these analyses, expressed as relative peak heights in contimeters in $\mathrm{Ta}$ ble 1, provide a semiquantitative basis for comparing the mineralogy of silt from dif- 
Table 2. Mineralogy of sand-size fraction from profundal sediments of 23 lakes.

\begin{tabular}{|c|c|c|c|c|c|c|}
\hline LAKE & $\begin{array}{c}\text { SAND } \\
\text { (\% clastic) }\end{array}$ & $\begin{array}{c}\text { QUARTZ } \\
(\%)\end{array}$ & $\begin{array}{c}\text { POTASH } \\
\text { FELDSPAR } \\
(\%)\end{array}$ & $\begin{array}{c}\text { PLAGIOCLASE } \\
\text { FELDSPAR } \\
(\%)\end{array}$ & $\begin{array}{l}\text { OPAQUE } \\
\text { MINERALS } \\
(\%)\end{array}$ & $\begin{array}{l}\text { OTHER } \\
(\%)\end{array}$ \\
\hline BIG & 2.1 & 46 & 18 & 31 & 4 & 1 \\
\hline IRON & 3.1 & 29 & 15 & 45 & 1 & 9 \\
\hline TROUT & 0.5 & 39 & 35 & 23 & & \\
\hline WILSON & 29.5 & 26 & 26 & 30 & 4 & 13 \\
\hline $0^{\prime}$ LEARY & 4.4 & 58 & 14 & 26 & $<1$ & 2 \\
\hline ARCO & 1.3 & 47 & 23 & 19 & 9 & 1 \\
\hline LINWOOD & 3.0 & 52 & 14 & 33 & 1 & $<1$ \\
\hline SPECTACLE & 5.1 & 53 & 16 & 28 & & \\
\hline GLADSTONE & 13.3 & 45 & 24 & 27 & 2 & 2 \\
\hline HAM & 8.8 & 55 & 17 & 22 & 3 & 3 \\
\hline REEDS & 2.9 & 46 & 11 & 41 & $<1$ & 1 \\
\hline ST. OLAF & 2.3 & 73 & 12 & 13 & $<1$ & 2 \\
\hline CHRISTMAS & 3.2 & 48 & 18 & 33 & 1 & $<1$ \\
\hline LOTUS & 2.0 & 51 & 12 & 31 & 3 & 1 \\
\hline LONG & 6.2 & 56 & 5 & 32 & 1 & 5 \\
\hline LITTLE PINE & 5.8 & 47 & 20 & 29 & 2 & 3 \\
\hline GROVE & 0.9 & 34 & 30 & 33 & 2 & 2 \\
\hline FISH & 4.3 & 38 & 19 & 42 & 2 & 0 \\
\hline SALLIE & 3.5 & 47 & 18 & 32 & 1 & 2 \\
\hline COWDRY & 1.0 & 46 & 23 & 23 & 7 & 1 \\
\hline SHETEK & 1.4 & 50 & 26 & 23 & $<1$ & $<1$ \\
\hline DEAD COON & 8.4 & 51 & 15 & 31 & 1 & 2 \\
\hline SALT & 3.6 & 41 & 16 & 38 & 4 & 2 \\
\hline
\end{tabular}

fercnt lake sediments. In most cases two distinct feldspar poaks were recognized in the $3.2 \AA$ region-one at $3.20 \AA$ and one at $3.18 \AA$. They probably represent orthoclase and plagioclase feldspar respectively.

Scmiquantitative analyses for mincralogy of the clay-size fraction $(<2 \mu)$ werc made on oriented glass-slide mounts by X-ray diffraction with Ni-filtered $\mathrm{Cu}-\mathrm{K} \alpha$ radiation, as for silt-sizc mincralogy. The clay minerals were oriented with c-axes porpondicular to the glass slide by pipetting a heavy slurry of clay in water onto the slide, allowing the clay minerals to settle out with a preferred orientation, and cvaporating the water. X-ray diffractograms were made on air-dried clay mounts and on the same mounts aftcr saturation with ethylene glycol to test for expansion of montmorillonitc. No attempt was made to separate the $7 \AA$ kaolinite and chlorite peaks. Representative patterns are presented in Fig. 5. Quantification of peak intensity is difficult owing. to the poor crystallinity of most samples. Some samples (e.g. Cedar Bog Pond, Fig. 5 ) appcar almost cntirely amorphous to Xradiation and produce no pcaks at the in- strument settings used. All samples were run at the same settings to producc comparable diffraction patterns. Relative intensities of mineral peaks in the clay-size fraction are given in Table 3 . The degrec of crystallinity, based on peak sharpness and intensity (Carroll 1970), is also given in relative terms.

Mineralogy of untreated samples was detormined by X-ray diffraction of randomly oricnted powdered samples mounted on glass slides: representative patterns are presented in Fig. 6. Semiquantitative rosults, expressed as peak height in centimeters for calcite, aragonite, dolomite, and quartz, are given in Table 1.

\section{Results}

The geographic patterns of organic, carbonate, and clastic materials in profundal sediments of Minnesota Lakes are shown in. Fig. 7.

Organic matter-Organic matter as loss on ignition in profundal sediments is greatest, though not invariably high, in lakes of the northern and eastern forested regions 


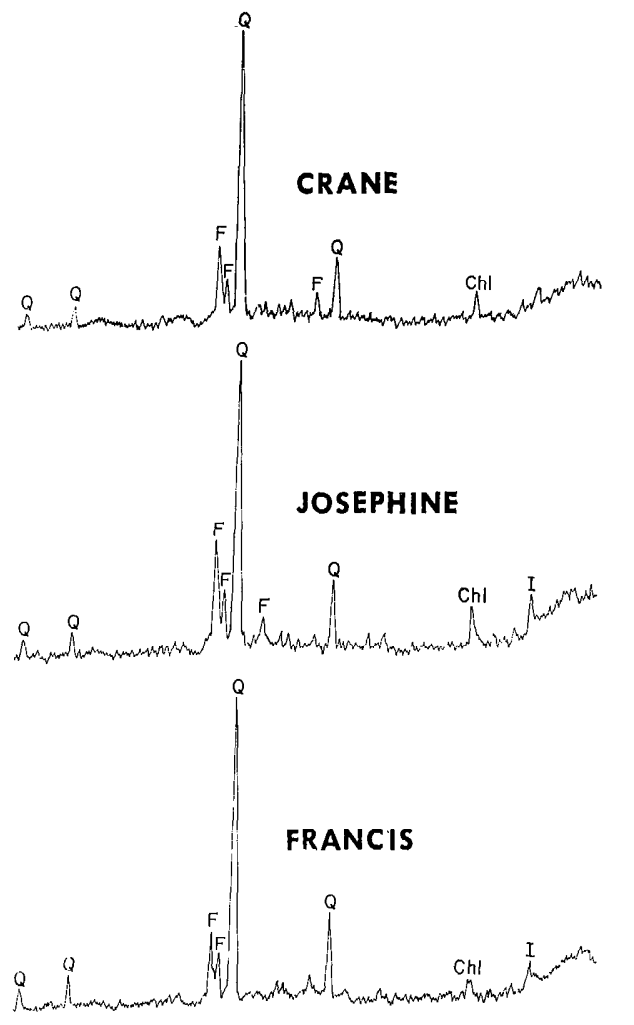

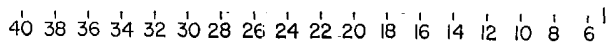
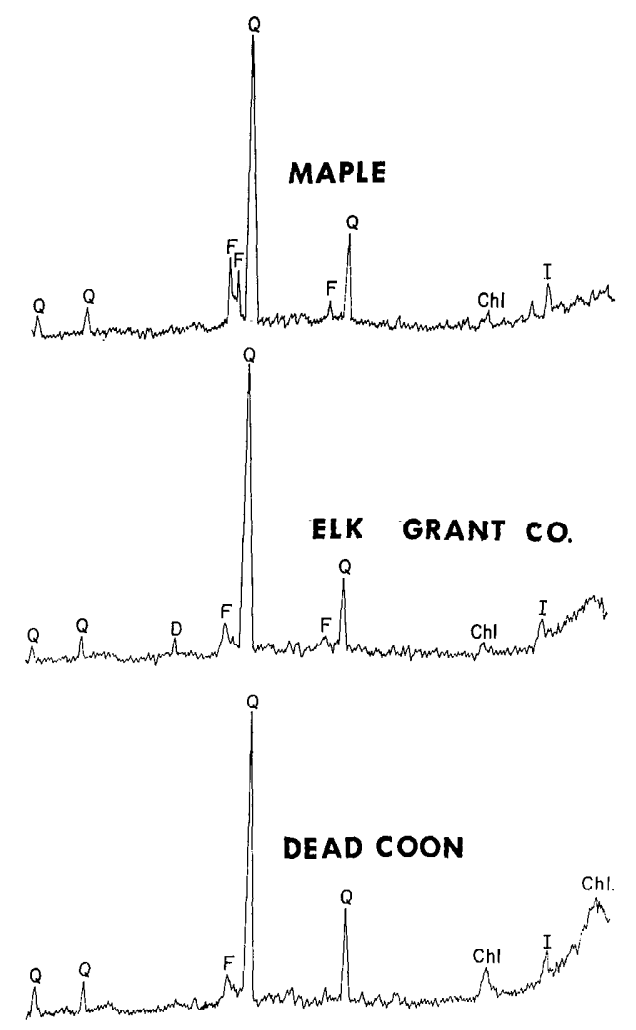

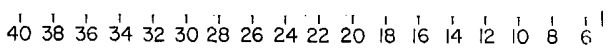

DEGREES $2 \theta$

Fig. 4. Representative X-ray diffraction patterns of silt-size fractions of profundal sediments of six lakes. Q-Quartz; F-feldspar; D-dolomite; I-illite; Chl一chlorite.

(Fig. 7A). In all the lakes it ranges from a high of $60 \%$ (Cedar Bog, east-central) to a low of $6.4 \%$ (Dead Coon, southwestern prairie) (Table 1; Figs. 7A and 8). The mean is $24.6 \%$ (SD 12.2). This corresponds to a mean organic-C (total-C minus carbonate-C) of $12.1 \pm 6.3 \%$, and a mean $i g$ nition loss : organic- $C$ ratio of $2.13 \pm 0.40$ (Fig. 3). When organic content is measured as percent total $\mathrm{N}$, most of these sediments have an organic- $C: N$ ratio of less than 10 on a weight percent basis, with a mean ratio of 8.9 (Fig. 9).

Carbonate-Profundal sediments in the central lakes may contain up to $46 \%$ carbonate as calculated from loss on ignition (Fig. 7B). Mean sedimentary carbonate in all the lakes is $16.3 \%$ (SD 14). Mean carbonate content in profundal scdiments from the 27 carbonate lakes (those with greater than $10 \%$ sedimentary carbonate) is $26.3 \%$. Sedimentary carbonate is restricted to those lakes with total cation concentrations in surface waters greater than $2 \mathrm{epm}$, which corresponds to $\mathrm{Ca}^{2+}$ concentrations $>1$ epm $(20 \mathrm{ppm})$ (Fig. 10). Theoretical saturation values of 22-24 ppm Ca ${ }^{2+}$ can be expected from the solubility of $\mathrm{CaCO}_{3}$ at $25^{\circ} \mathrm{C}$ and $\mathrm{CO}_{2}$ concentrations in the range 0.033 0.044\% (Hutchinson 1957).

The zero-carbonate baseline in Figs. 8 and 10, at about $3 \%$ carbonate, represents sediments with no carbonate and is presumably duc to loss of water from clay lattices 


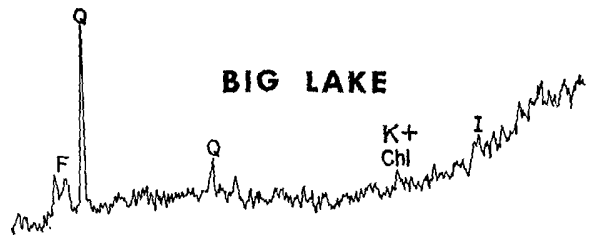

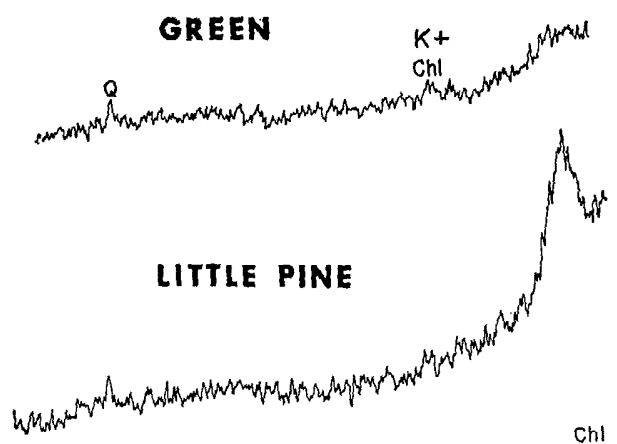

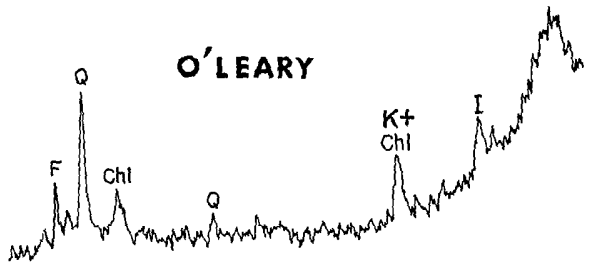

CEDAR BOG

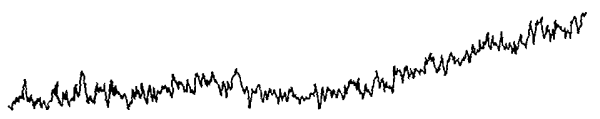

BEAVER

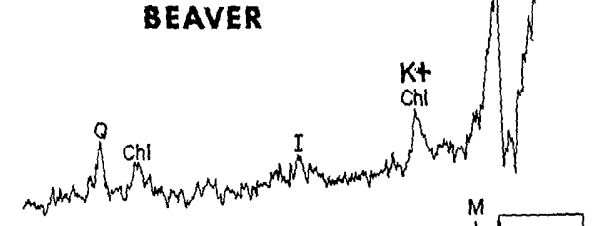

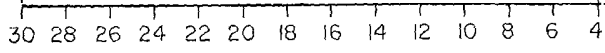

DEGRES $2 \theta$

Fig. 5. Representative X-ray diffraction patterns of clay-size fractions, solvated with ethylene gylcol, of profundal sediments of eight lakes. Q-Quartz; F-feldspar; I-illite; M-montmorillonite; Chlchlorite; K-kaolinite.

(Dean 1974). The value of about 3\% applies only to those samples lacking carbonate. If we assume that those sediments with $550^{\circ}-1,000^{\circ} \mathrm{C}$ ignition losses $<5 \%$ contain no carbonate, then the mean carbonate contont of sediments in all 46 lakes would be lowered slightly-to about $15 \%$. The lakes with profundal sediments lacking carbonate include all group 1 lakes, and four of the more dilute group 2 lakes with relatively high organic scdiments, all located on highly leached outwash of the Anoka Sand Plain (Sims and Morey 1972).

Most of the carbonate is in the form of low- $\mathrm{Mg}$ calcite, although minor amounts of high-Mg calcite, dolomite, and aragonite were detected by X-ray diffraction (Table 1, Figs. 6 and 11). The dolomite occurs in lake sediments in southern and western Minnesota (Table 1). In addition, X-ray 
Table 3. Mineralogy of clay-size $(<2 \mu)$ fraction from profundal sediments of all 46 lakes.

\begin{tabular}{|c|c|c|c|c|c|c|c|c|c|c|c|c|}
\hline \multirow[b]{2}{*}{ LAKE } & \multirow{2}{*}{\multicolumn{2}{|c|}{$\begin{array}{l}\text { Degree of } \\
\text { crysta11- } \\
\text { inity * }\end{array}$}} & \multirow{2}{*}{\multicolumn{2}{|c|}{$\begin{array}{c}\text { ILLITE } \\
(10 \AA) \\
\text { untr. }^{+} \text {g1y. }\end{array}$}} & \multirow{2}{*}{\multicolumn{2}{|c|}{$\begin{array}{l}\text { KAOLINITE + } \\
\text { CHLORITE } \\
(7 \AA) \\
\text { untr. gly. }\end{array}$}} & \multicolumn{2}{|c|}{$\begin{array}{l}\text { QUARTZ } \\
(3.35 \AA)\end{array}$} & \multicolumn{2}{|c|}{$\begin{array}{l}\text { FELDSPAR } \\
(3.2 \AA)\end{array}$} & \multicolumn{2}{|c|}{$\begin{array}{c}12-14 \AA \\
\text { peak }\end{array}$} \\
\hline & & & & & & & untr. & gly. & untr. & gly. & untr. & gly. \\
\hline Big & poor & & $\operatorname{tr} \xi$ & $\operatorname{tr}$ & $\mathrm{tr}$ & $\operatorname{tr}$ & ++ & ++ & + & + & A & A \\
\hline Iron & poor & & 0 & 0 & 0 & 0 & $\operatorname{tr}$ & 0 & 0 & 0 & $A$ & A \\
\hline Mounta in & fair & & + & + & + & + & + & + & $\operatorname{tr}$ & $\operatorname{tr}$ & $A$ & A \\
\hline Trout & poor & & 0 & 0 & $\operatorname{tr}$ & $\operatorname{tr}$ & $\operatorname{tr}$ & $\operatorname{tr}$ & 0 & 0 & A & A \\
\hline Clearwater & fair & & + & + & + & + & + & + & 0 & 0 & $\mathrm{P}$ & A \\
\hline Wilson. & poor & & $\operatorname{tr}$ & 0 & $\operatorname{tr}$ & $\operatorname{tr}$ & $\operatorname{tr}$ & $\operatorname{tr}$ & $\operatorname{tr}$ & $\operatorname{tr}$ & $P^{-}$ & A \\
\hline Sand Point & poor & & 0 & 0 & $\operatorname{tr}$ & 0 & $\operatorname{tr}$ & $\operatorname{tr}$ & $\operatorname{tr}$ & $\operatorname{tr}$ & $\mathrm{P}^{-}$ & A \\
\hline Kimbal1 & poor & $F$ & $\operatorname{tr}$ & 0 & 0 & 0 & tr & tr & tr & tr & $\mathrm{p}$ & $\ddot{A}$ \\
\hline Crane & fair & 으 & $\operatorname{tr}$ & $\operatorname{tr}$ & + & + & + & + & $\operatorname{tr}$ & $\operatorname{tr}$ & $P_{+}$ & A \\
\hline 0'Leary & good & $\overrightarrow{3}$ & + & + & + & + & + & + & + & + & $\mathrm{P}^{+}$ & $17 \AA$ \\
\hline Josephine & fair & 은 & $\operatorname{tr}$ & $\operatorname{tr}$ & + & + & + & + & + & + & $\mathrm{P}^{-}$ & $\mathrm{p}^{-}$ \\
\hline Arco & fair & $\overline{0}$ & $\operatorname{tr}$ & $\operatorname{tr}$ & + & + & + & + & + & + & $\mathrm{P}^{-}$ & $A$ \\
\hline Deming & fair & & $\operatorname{tr}$ & $\operatorname{tr}$ & + & + & + & + & + & + & $\mathrm{P}^{-}$ & A \\
\hline Farquar & poor & & + & + & + & + & + & + & $\operatorname{tr}$ & $\operatorname{tr}$ & A & A \\
\hline George & poor & & $\operatorname{tr}$ & $\operatorname{tr}$ & 0 & 0 & $\operatorname{tr}$ & $\operatorname{tr}$ & $\operatorname{tr}$ & $\operatorname{tr}$ & $\mathrm{P}$ & A \\
\hline Linwood & poor & & 0 & 0 & 0 & 0 & $\operatorname{tr}$ & $\operatorname{tr}$ & 0 & 0 & $\mathrm{P}^{-}$ & A \\
\hline Cedar Bog & poor & & 0 & 0 & 0 & 0 & 0 & 0 & 0 & 0 & 0 & 0 \\
\hline Spectacle & poor & & $\operatorname{tr}$ & $\operatorname{tr}$ & $\operatorname{tr}$ & 0 & + & + & $\operatorname{tr}$ & $\operatorname{tr}$ & $\mathrm{P}^{-}$ & A \\
\hline Gladstone & poor & & 0 & 0 & $\operatorname{tr}$ & 0 & $\operatorname{tr}$ & tr & 0 & 0 & $\mathrm{P}^{-}$ & A \\
\hline Ham & poor & & 0 & 0 & 0 & 0 & 0 & $\operatorname{tr}$ & 0 & 0 & A & A \\
\hline Green & poor & & 0 & 0 & $\operatorname{tr}$ & $\operatorname{tr}$ & $\operatorname{tr}$ & $\operatorname{tr}$ & 0 & 0 & P & $17 \AA$ \\
\hline Reeds & good & & + & + & + & + & + & + & $\operatorname{tr}$ & $\operatorname{tr}$ & $P$ & $\mathrm{P}$ \\
\hline Francis & good & & + & + & + & + & + & + & + & + & $P$ & $17 \AA$ \\
\hline Nokay & poor & & $\operatorname{tr}$ & 0 & $\operatorname{tr}$ & $\operatorname{tr}$ & $\operatorname{tr}$ & $\operatorname{tr}$ & 0 & 0 & $A_{+}$ & A \\
\hline Beaver & exce1. & & ++ & & + & & + & & $\operatorname{tr}$ & & $p^{+}$ & \\
\hline Moose & good & & + & 0 & + & + & + & + & $\operatorname{tr}$ & $\operatorname{tr}$ & $\mathrm{P}$ & $\mathrm{P}$ \\
\hline Bal1 Club & poor & & 0 & 0 & 0 & 0 & + & $\operatorname{tr}$ & 0 & & A & A \\
\hline St. $01 a f$ & good & & + & + & + & + & + & + & + & $\operatorname{tr}$ & $P_{-}$ & $\mathrm{P}$ \\
\hline Christmas & fair & $\mathbf{N}$ & + & $\operatorname{tr}$ & + & $\operatorname{tr}$ & + & + & + & $\operatorname{tr}$ & $\mathrm{P}_{+}^{-}$ & A \\
\hline Lotus & fair & 은 & + & + & + & + & + & + & + & + & $\mathrm{p}_{+}^{+}$ & $17 \AA$ \\
\hline Clear & good & & + & $\operatorname{tr}$ & + & $\operatorname{tr}$ & + & + & + & $\operatorname{tr}$ & $p^{+}$ & A \\
\hline Long & fair & 은 & + & + & + & + & + & + & + & + & $\mathrm{P}$ & $\mathrm{p}$ \\
\hline Elk & poor & & 0 & 0 & 0 & 0 & $\operatorname{tr}$ & $\operatorname{tr}$ & 0 & 0 & A & A \\
\hline Itasca & poor & & 0 & 0 & 0 & 0 & tr & $\operatorname{tr}$ & 0 & 0 & A & A \\
\hline Little Pine & fair & & $\operatorname{tr}$ & $\operatorname{tr}$ & 0 & $\operatorname{tr}$ & + & + & + & $\operatorname{tr}$ & P & $\mathrm{P}$ \\
\hline Grove & fair & & + & $\operatorname{tr}$ & + & 0 & + & + & $\operatorname{tr}$ & 0 & $A$ & A \\
\hline Fish & fair & & + & $\operatorname{tr}$ & + & $\operatorname{tr}$ & + & + & 0 & 0 & $A$ & A \\
\hline Sallie & fair & & + & + & + & + & + & + & + & $\operatorname{tr}$ & $\mathrm{P}$ & $\mathrm{p}$ \\
\hline Cowdry & poor & & $\operatorname{tr}$ & 0 & $\operatorname{tr}$ & 0 & + & tr & $\operatorname{tr}$ & 0 & A & A \\
\hline Maple & poor & & + & + & + & + & + & + & + & + & $A$ & A \\
\hline Mina & poor & & $\operatorname{tr}$ & $\operatorname{tr}$ & $\operatorname{tr}$ & tr & + & + & $\operatorname{tr}$ & 0 & A & A \\
\hline Shetek & excel. & & + & + & $t+$ & + & $T$ & + & $\operatorname{tr}$ & $\operatorname{tr}$ & A & $\bar{P}$ \\
\hline Big Kandiyohi & excel. & $m$ & + & + & + & + & + & + & $\operatorname{tr}$ & $\operatorname{tr}$ & P & P \\
\hline Elk (Grant Co.) & excel. & (i & ++ & $+t$ & ++ & $+t$ & + & + & $\operatorname{tr}$ & $\operatorname{tr}$ & $P$ & $P$ \\
\hline Dead Coon & excel. & 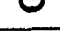 & ++ & ++ & ++ & ++ & + & + & + & + & $P$ & $\mathrm{P}$ \\
\hline Sa7t & excel. & + & + & + & + & + & + & + & + & + & $P$ & $\bar{P}$ \\
\hline
\end{tabular}

* Relative degree of crystallinity for these samples only.

+ Untreated sample, dry on slide.

$\neq$ Sample after treatment with ethylene glycol.

5 Peak intensities: $++=$ very wel1 defined peak; $+=$ well defined peak; $t r=$ trace or peak very poorly defined; $0=$ no peak above background; $P=$ well defined $12-14 \AA ; A=$ only low angle background slope at $12-14 \AA$. 


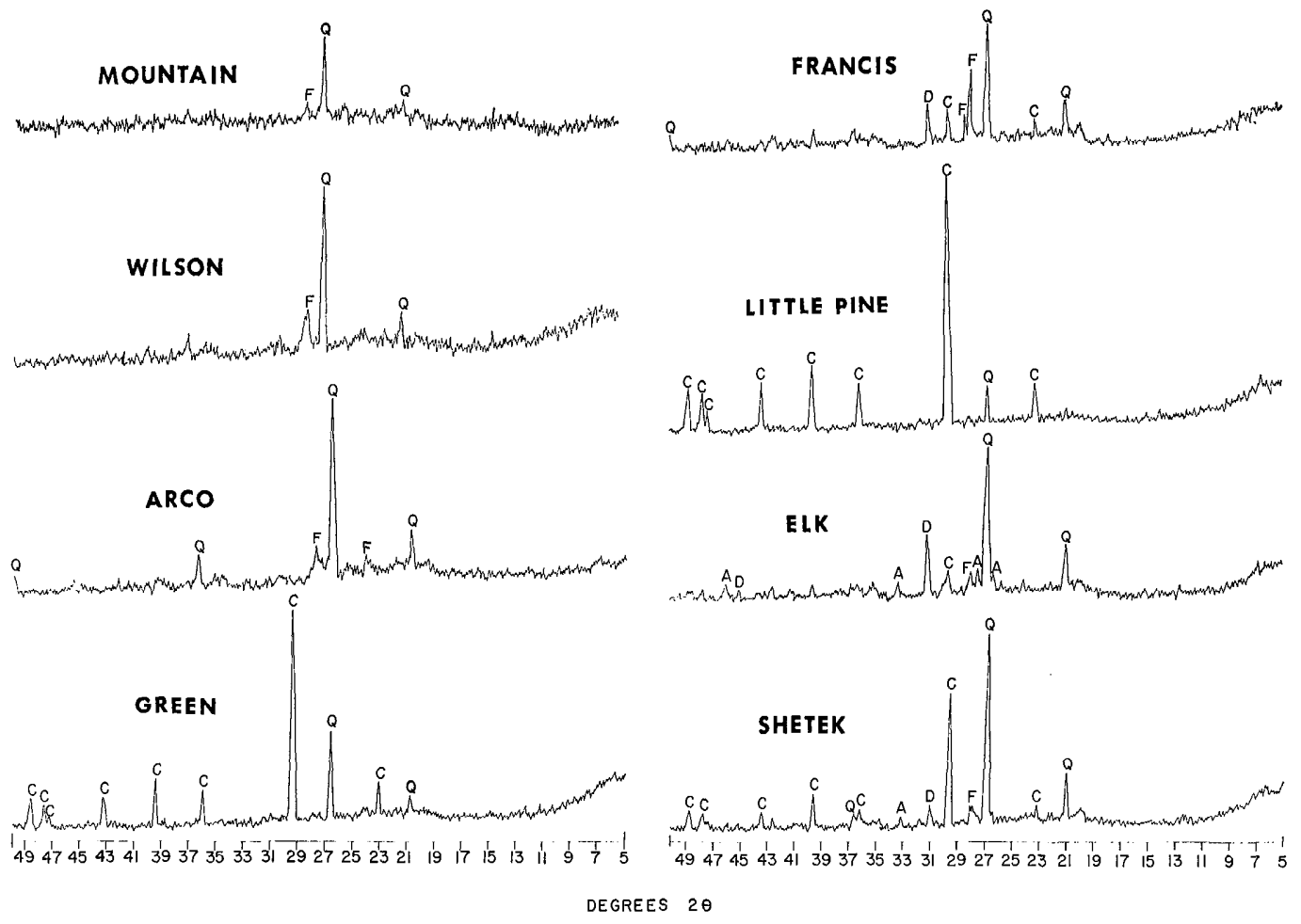

Fig. 6. Representative X-ray diffraction patterns of untreated samples of profundal sediments of eight lakcs. Q- Quartz; F-feldspar; C-calcite; D-dolomitc; A-aragonitc.

diffraction patterns of sediments from several of these lakes (e.g. Francis, Bcaver, Fish, Cowdry, Sallic, Dead Coon, and Salt) exhibit shoulders at about $29.7^{\circ} 2 \theta$ on the main calcite peak $\left(29.4^{\circ}\right)$, suggesting that some high-Mg calcite may be present. Some dolomite in group 3 and high-carbonate group 2 lakes may therefore be a diagenetic product of high-Mg calcite, as suggestcd by Müller ct al. (1972), or it may be detrital, derived from Paleozoic dolostones. Müller et al. (1972) found primary aragonite only in lakes with a $\mathrm{Mg}: \mathrm{Ca}$ ratio in the water of $>12$. The small amounts of aragonite in Minnesota lakes are probably derived from molluse shells because the $M g: C a$ in waters from these lakes is generally $<2$.

The lake with the most instructive carbonate mineralogy is Elk Lake, Grant Co. (No. 44). Its sediments contain more do- lomite than any of the other lakes (Table 1, Fig. 6). Although Elk is not the most saline of the lakes studied, it is the only one with a high $\mathrm{Mg}: \mathrm{Ca}$ ratio (7.7) in the water. $\mathrm{X}$-ray diffraction patterns of its sediments commonly show two distinct calcite peaks, one at $29.4^{\circ} 2 \theta$ (low-Mg calcite) and one at about $29.7^{\circ}$ (high-Mg calcitc) (Fig. 11). Figurc 12 shows that Elk Lake sediments (point marked 44) contain much more total Mg than any other lake sediments, but it is largely in dolomite which is not soluble in $1.0 \mathrm{~N}$ ammonium acetate at $\mathrm{pH} 5.2$ (Wangersky and Joensuu 1967). According to Müller ct al. (1972), diagenetic dolomite is found in lakes with high-Mg calcite as a primary carbonate mineral and a $M g: C a$ ratio in the water of $>7$.

For most sediments high in carbonate (i.e. $>10 \%$ ), about half of the $\mathrm{Mg}$ is soluble in acid ammonium acctatc, presumably in 

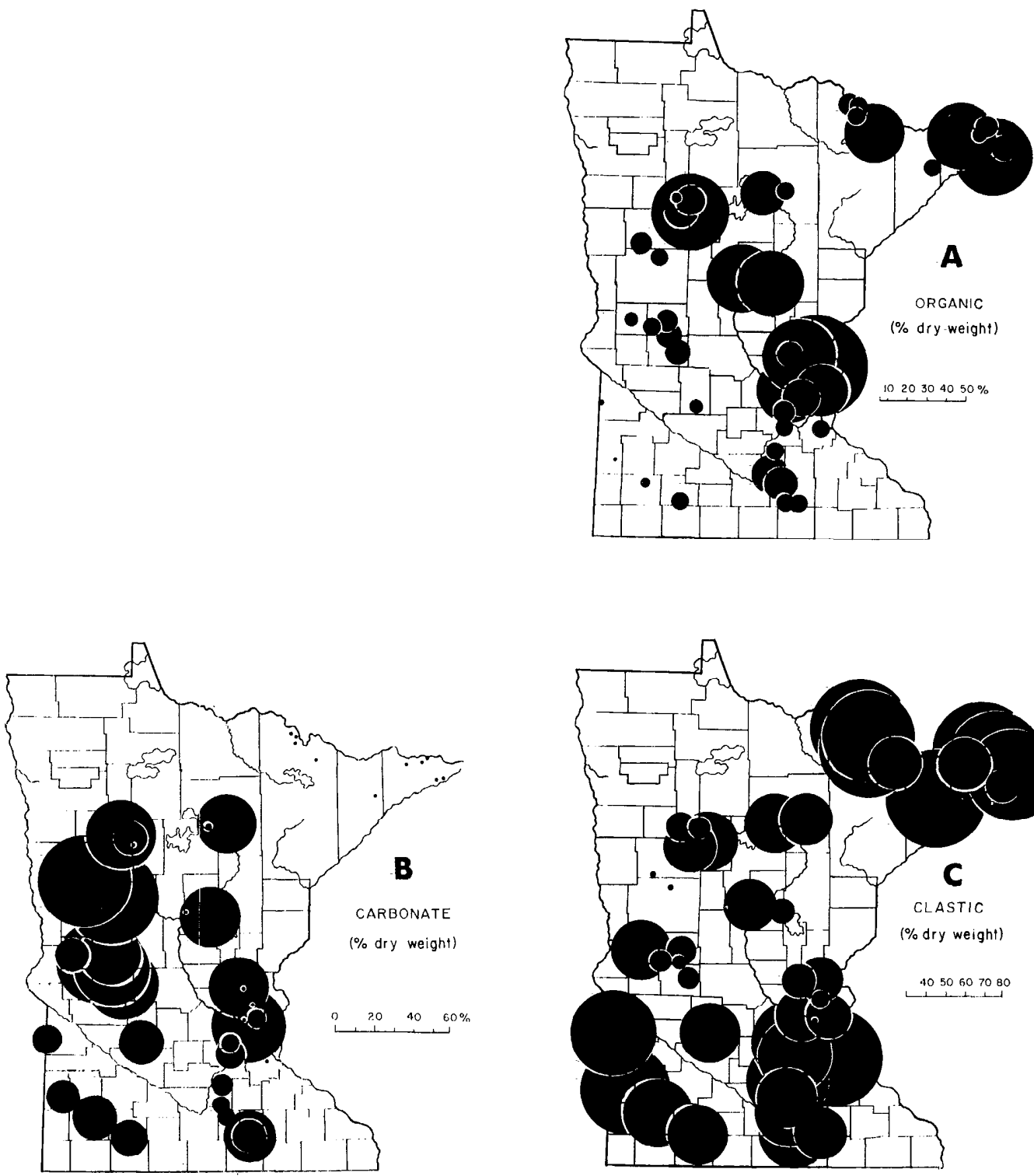

Fig. 7. Maps showing distribution of percent organic matter (A), percent carbonate (B), and percent clastic material (C) in profundal sediments of all 46 lakes. All values calculated from loss on ignition at $550^{\circ}$ and $1,000^{\circ} \mathrm{C}$ ( see text). Note that minimum values for percent clastic and percent organic matter are $35 \%$ and $6 \%$.

solid solution for $\mathrm{Ca}$ in the calcite structure (Fig. 12). The total soluble Mg, expressed as percent dry weight, increases with increasing $M g: C a$ ratio of the water (Fig. 13). In those lakes which precipitate the most calcium carbonate, i.e. the group 3 and high-carbonate group 2 lakes, the proportion of Mg coprecipitated with calcite, expressed as percent calcium carbonate, increases with increasing $\mathrm{Mg}: \mathrm{Ca}$ ratio in the lake water (Fig. 14). In other words, in lakes of western and southwestern Minnesota, the $M g$ : 


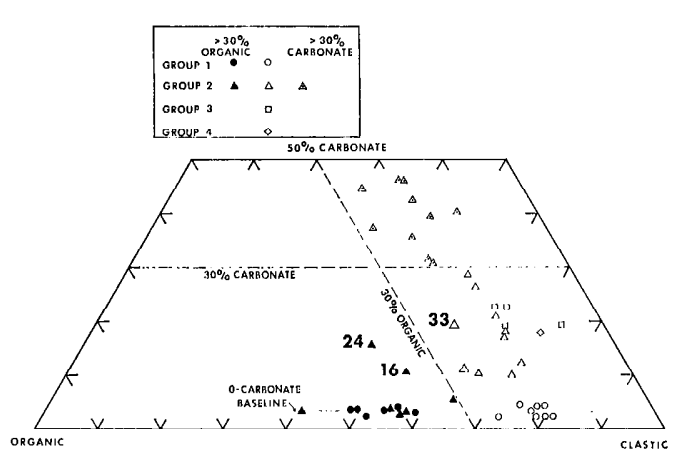

Fig. 8. Trilinear plot of carbonate, organic, and clastic fractions in profundal sediments of all 46 lakes. The 0-carbonate refers to ignition loss between $550-1,000^{\circ} \mathrm{C}$ of sediments containing no carbonate minerals. Numbers adjacent to points refer to lakes (Fig. 2) specifically discussed in text.

$\mathrm{Ca}$ ratio of sedimentary calcium carbonate increases with increasing $\mathrm{Mg}: \mathrm{Ca}$ in the water. Figures 13 and 14 also show that for low-carbonate group 2 lakes (sedimentary carbonate $<30 \% ; \overrightarrow{M g}: C a$ ratio in water generally $<1.0$ ), there is no apparent rela-

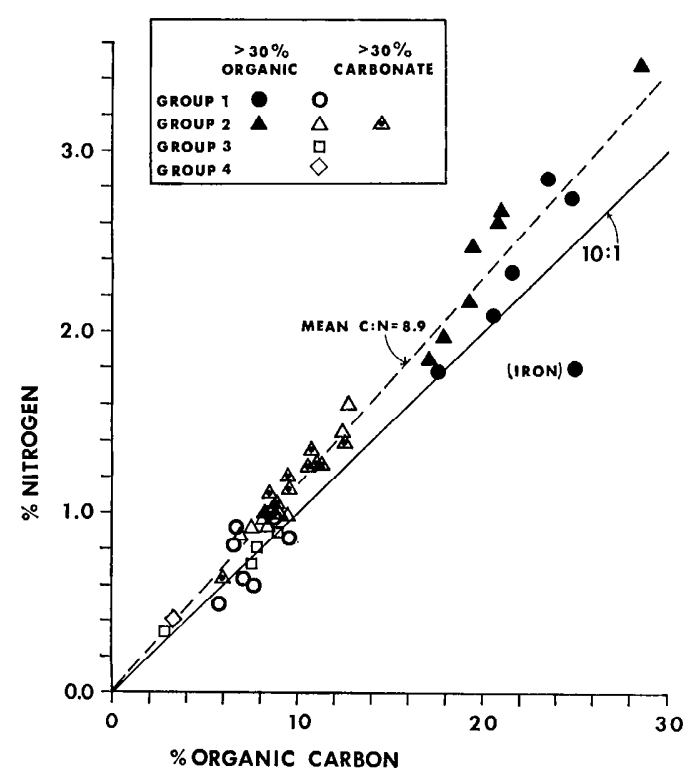

Fig. 9. Scatter plot of weight percent nitrogen and weight percent organic carbon in profundal sediments from all 46 lakes.

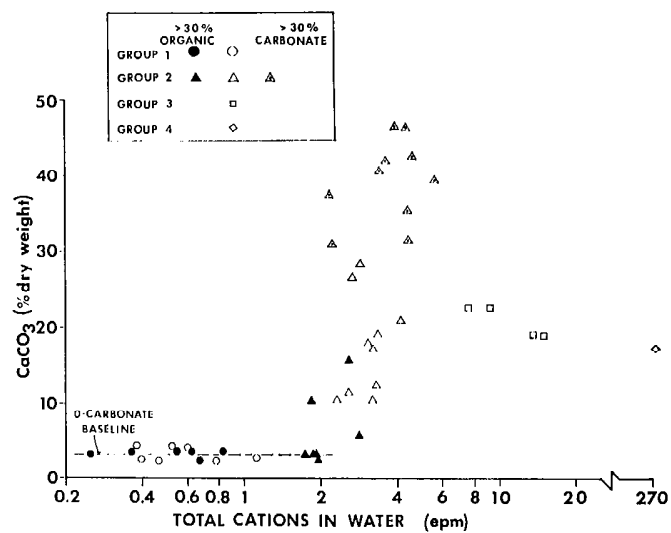

Fig. 10. Semilogarithmic plot of carbonate by ignition loss in profundal sediments and total cations in surface waters from all 46 lakes.

tionship between soluble $\mathrm{Mg}$ and the $\mathrm{Mg}$ : $\mathrm{Ca}$ ratio of the water. However, some of these lakes contain considerable soluble Mg. If this $\mathrm{Mg}$ is present in solid solution for $\mathrm{Ca}$ in calcite, then these lakes must have sedimentary carbonates with a higher $M g$ : $\mathrm{Ca}$ ratio than those of group 3 and high-carbonate group 2. This is unlikely because the $M g$ : $C a$ ratios in these waters are much lower than in the western and southwestern lakcs. Presumably the soluble $\mathrm{Mg}$ of the low-carbonatc group 2 lakes is adsorbed on

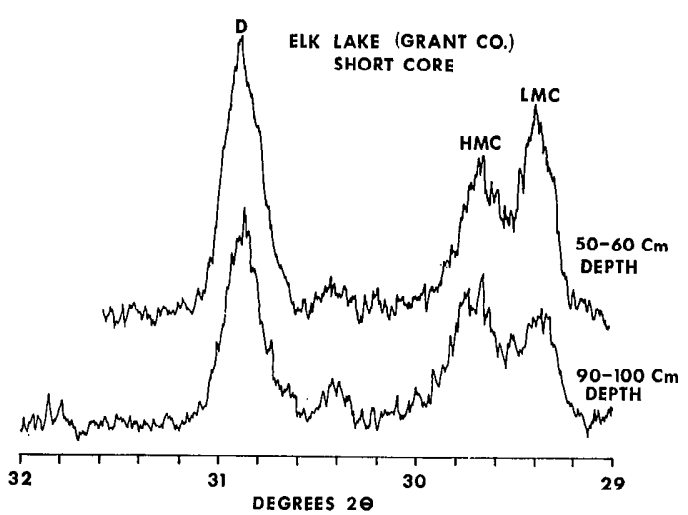

Fig. 11. X-ray diffraction patterns from slow $\left(0.4^{\circ} 2 \theta \mathrm{min}^{-1}\right)$ scans between $29^{\circ}$ and $32^{\circ} 2 \theta$ of sediment samples from two depths in short core from Elk Lake, Grant Co. HMC, LMC, and D refer to peaks for high-Mg calcite, low-Mg calcite, and dolomite. 


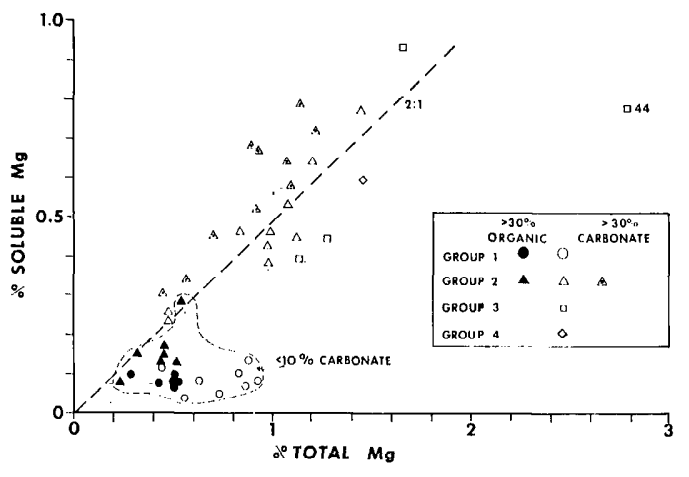

Fig. 12. Scatter plot of $\mathrm{Mg}$ soluble in 1.0 $\mathrm{N}$ ammonium acetate ( $\mathrm{pH} 5.2$ ) and total $\mathrm{Mg}$ in profundal scdiments from all 46 lakes.

clays, bccause soluble $\mathrm{Mg}$ is not high in lakes with high sedimentary organic matter (Table 1).

Clastic material-The clastic fraction is defincd as the noncarbonate, inorganic mincral material in a sample. Lake sediments with the largest clastic fractions occur in northeastern and southwestern Minnesota (Fig. 7C). Lake sediments from central Minnesota contain relatively low clastic fractions, as a result of dilution by carbonate and organic matter (Figs. 7A and 7B). The range of clastic matcrial is from $35-81 \%$, with a mean of $58.9 \%$ (SD 13.7).

After destruction of the organic and carbonate components, the clastic fraction was further subdivided into sand, silt, and clay

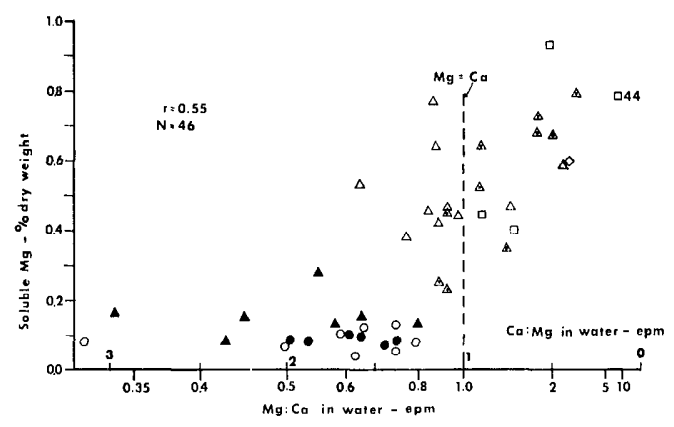

Fig. 13. Scatter plot of sedimentary Mg soluble in $1.0 \mathrm{~N}$ ammonium acctate (pH 5.2 ), expressed as percont dry weight, and $M g: C a$ ratio in surface waters from all 46 lakes. (Source of data-Gorham et al. in prep.)

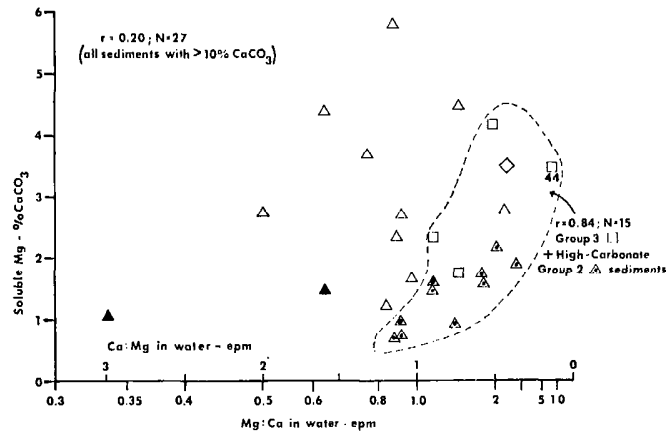

Fig. 14. Scatter plot of sedimentary $\mathrm{Mg}$ soluble in $1.0 \mathrm{~N}$ ammonium acetate (pII 5.2), expressed as percent $\mathrm{CaCO}_{3}$, and $M g: C a$ ratio in surface waters from all 46 lakes.

fractions. Significant proportions of sand occur in very few profundal sediments and exceed $10 \%$ dry weight only in three northern lakes. Silt and clay arc present in about equal proportions in most samples. Mean values and standard deviations for sand, silt, and clay, expressed as percent dry weight, are $2.9 \%$ ( \pm 4.2$), 23.4 \%$ ( \pm 8.1 ), and

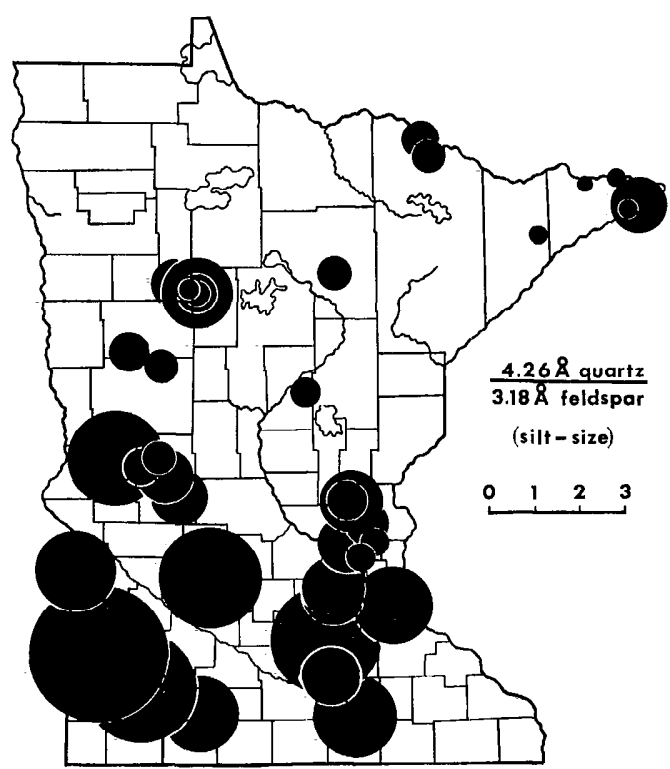

Fig. 15. Map showing ratio of $4.26 \AA$ quartz poak height to $3.18 \AA$ feldspar peak height from $\mathrm{X}$ ray diffraction traces of silt-size materials in profundal sediments from 38 lakes. 
$22.3 \%( \pm 8.4)$. Expressed as perccnt clastic material, the respcctive means are $7.3 \%$, $47.6 \%$, and $46.8 \%$.

The sand-size fraction (Table 2) is characterized by a predominance of quartz (46.9\%), with lesser amounts of plagioclase $(29.8 \%)$ and orthoclase (18.6\%). No regional variations exist in the proportions of the'se minerals.

The silt-size fraction is also dominated by quartz and, to a lesser extent, plagioclase and orthoclase feldspar (Table 1 and Fig. 4). Most X-ray diffractograms exhibit trace or moderate low angle peaks for clay minerals as well; most common are illite $(10 \AA)$ and kaolinite + chlorite $(7 \AA)$. Figure 15 illustrates a marked northcast to southwest change in the mineralogy of the silt-size fraction. On the basis of peakheight ratios, the abundance of quartz relative to feldspar is grcatest in group 3 scdiments of the arid southwest, and lowest in group 1 sediments of the humid northeast.

Most X-ray diffractograms of clay-size matcrial are characterized by a $3.2 \AA$ feldspar pcak, a $3.35 \AA$ quartz pcak, $3.5 \AA$ and $7 \AA$ kaolinite + chlorite pcaks, and a $10 \AA$ illite peak (Table 3; Fig. 5). For some samples it was possible to distinguish between the $3.57 \AA$ kaolinite poak and the $3.52 \AA$ chlorite peak, but for most the $3.5 \AA$ region yiclded a broad single peak; no attempt was made to separate the two peaks by heating. In some samples, the presence of montmorillonite is indicated by the development of a $17 \AA$ peak from $12-14 \AA$ mixcd-layer clays after treatment with ethylene glycol.

The values in Table 3 show no woll defincd regional differences in clay mineralogy, although there is an apparent incrcase in all clay minerals in sediments from group 3 lakes in southwestern Minnesota. However, values for percent clay in Table 1 show no regional variation in the amount of clay-size material. Therofore, the apparent increase in clay mincrals in the southwestern lakc sediments is due not to an increase in actual clay mineral content, but to a higher degrec of crystallinity of the clay-size fraction, resulting in stronger $\mathrm{X}$ ray diffraction peaks. Most of the clay-size matcrial in sediments from group 1 and group 2 lakes in northeastern and central Minnesota appears to be X-ray amorphous. Ross and Kerr (1931) have suggested that the name allophane be applied to all X-ray amorphous clay minerals, regardless of composition. Most of this allophane is probably opaline silica derived from diatom remains. However, the fact that the claycrystallinity gradient corresponds to the regional climatic gradient of the state suggests that climate has caused a greater alteration of clays in the northeast than in the southwest.

\section{Discussion}

Lake grouping-Figures 2 and 8 show that the geographic groups of the lakes defined initially on the basis of water chemistry (Gorham et al. in prep.) can also be differentiated on the basis of profundal sediment chemistry and mineralogy. The sediments of dilute group 1 lakes on noncalcareous drift in northeastcrn Minncsota arc characterized by an absence of carbonate mincrals. Profundal sediments of the deep $(>10 \mathrm{~m})$ group 1 lakes contain much less organic material (16-21\% loss on ignition at $550^{\circ} \mathrm{C}$ ) than those of the shallow group 1 lakes (38-47\% loss on ignition). Throe very small outlying group 1 lakes in Hubbard Co. (Fig. 2; Table 1) are also rich in organic matter despite depths of 7.6, 11.6, and $17.0 \mathrm{~m}$. All threc have unusually high ratios of depth to area and are sheltered from wind mixing; as a result, they do not undergo normal mixing and may remain stratificd for several years.

Group 2 lakes, of intermediatc ionic concentration, have the most diverse sediments, due mainly to variation in the amount of precipitated calcium carbonate. They arc distinguished from group 1 lakes by the presence of carbonate minerals in profundal sediments, except in four of tho most dilute group 2 lakes with highly organic scdiments $\left(>39 \%\right.$ loss on ignition at $550^{\circ} \mathrm{C}$ ). Waters of these high-organic group 2 lakes commonly have $\mathrm{Ca}^{2+}$ concentrations greater than $1.0 \mathrm{cpm}(20 \mathrm{ppm})$, a level sufficient 
to precipitate $\mathrm{CaCO}_{3}$ in other dilute, loworganic group 2 lakes. For example, George, Cedar Bog, Spcetacle, Ham, and Green are five relatively dilute group 2 lakes on the Anoka Sand Plain with very similar water chemistries (Fig. 2). George, Cedar Bog, and Spectacle contain $>39 \%$ organic matter and no carbonate, whereas Ham and Green contain 28 and $21 \%$ organic matter and 37 and $30 \%$ carbonate. Decomposition of organic matter in the profundal zone of the high-organic group 2 lakes apparently generates enough $\mathrm{CO}_{2}$ to redissolve any calcium carbonate that may have formed. Two other dilute group 2 lakes, Nokay and Linwood (Fig. 2), with slightly less organic matter (38\% and $35 \%$ ), contain $16 \%$ and $10 \%$ carbonate in their profundal sediments. These two lakes are transitional between the carbonate-free, high-organic group 2 lakes and those intermediate in both components.

Sedimcnts of intcrmediate group 2 lakes, containing $10-30 \%$ organic matter and 10 $30 \%$ carbonate, also tend to be intermediate in dissolved ionic concentration within group 2 lakes. With two exceptions, Moose and Elk (Clearwater Co.) (Fig. 2), intermediate group 2 lakes are in south-central and southwestern Minnesota, where evaporation exceeds precipitation, so that dissolved salts become concentrated particularly during summer. Calcium and alkalinity concentrations in these lakes are generally greater than 1.0 and $2.0 \mathrm{epm}$ and are greater than 2.0 and $3.0 \mathrm{epm}$ during summer (Gorham et al. in prep.; Bright 1968). Elk Lake (Clearwater Co., No. 33 in Fig. 2) is in west-central Minnesota, an area dominated by high-carbonate group 2 lakcs, although it classifies with the southcentral and southeastern intermediate group 2 lakes. This lake has a maximum depth of nearly $30 \mathrm{~m}$; it is the second deepest carbonate lake studied. The profundal sediments at this depth have a carbonate content of only $19 \%$, which would classify Elk as an intermediate group 2 lake. However, sediments from five other stations in Elk Lake, ranging from $11-24 \mathrm{~m}$ in depth, contain 24-70\% $\mathrm{CaCO}_{3}$, with $\mathrm{CaCO}_{3}$ con- tent roughly proportional to depth. Most sediments in this lake would place it as a high-carbonate $(>30 \%)$ group 2 lake, and indeed the surface waters commonly have $\mathrm{Ca}^{2+}$ and alkalinity concentrations of about 2 and 3 epm (Megard 1968; Gorham et al. in prep.). Elk Lake, like nearby Itasca and Long Lakes (Megard 1968), undoubtedly precipitates large quantities of calcium carbonate during summer, but dissolution of carbonate in the deeper waters of the lake has greatly reduced the amount of carbonate being incorporated into deeper profundal sediments. Moose Lake, the other geographic exception in the intermediate group 2 category, has $28.3 \%$ carbonate and therefore also comes close to being classificd with high-carbonate group 2 lakes.

The high carbonate $(>30 \%)$ group 2 lakes are all in west-central Minnesota (Fig. 2 ). They generally contain less than $30 \%$ organic matter and arc also characterizcd by inactive outlets and concentrations of dissolved $\mathrm{Ca}^{2+}$ and alkalinity in excess of 2.0 and $3.0 \mathrm{epm}$. Detailed studies by $\mathrm{Me}-$ gard (1968) on the precipitation of $\mathrm{CaCO}_{3}$ in two typical high-carbonate group 2 lakes (Long and Itasca) showed that their surface waters are supersaturated with respect to calcite during summer and precipitate large quantities of calcite. Megard also found a close correlation between dissolved $\mathrm{Ca}^{2+}$ and alkalinity depletion (presumably as precipitated $\mathrm{CaCO}_{3}$ ) and photosynthetic carbon assimilation in six typical high-carbonate group 2 lakes. For the six lakes, an average of 1 mole of $\mathrm{Ca}^{2+}$ was removed for evcry 4 moles of carbon fixed by phytoplankton photosynthesis. Because of the high rate of calcite precipitation, the highcarbonate group 2 lakes generally have ratios of dissolved $\mathrm{Mg}: \mathrm{Ca}>1.0$ (Figs. 13 and 14).

Group 3 lakes occupy shallow depressions in thin, gypsum-bearing glacial drift in the western and southwestern prairie regions. Dissolved salts in the waters of these arid regions are more concentrated than in those of group 1 or group 2 lakes; they are rich in dissolved magnesium, calcium, sulfate, and bicarbonate. Profundal sediments from 
group 3 lakes resemble those of intermediate group 2 lakes (Fig. 8), but are distinguished from them by the excellent degree of crystallinity of the clay-size fraction (Table 3 ), by their low organic content ( $<16 \%$ loss on ignition at $550^{\circ} \mathrm{C}$ : Fig. 7A), and by a strong predominance of the clastic fraction (62-75\%: Fig. 7C). The high $\mathrm{Mg}^{2+}$ concentration in group 3 lake watcrs is reflected by relatively high $\mathrm{Mg}$ concentrations in the sediments, presumably due to coprecipitation with calcite (Figs. 12-14). The percentage of carbonate in group 3 lake sediments $(17-26 \%)$ is less than in high-carbonate group 2 lake sediments (Figs. 8 and 10), despite higher concentrations of $\mathrm{Ca}^{2+}$ and alkalinity in the waters of group 3 lakes. This anomaly presumably reflects dilution by high silting in these shallow prairie lakes.

A rare fourth group of lakes, characterized by very saline waters (conductivity $>7,000 \mu$ mhos $\mathrm{cm}^{-1}$ at $25^{\circ} \mathrm{C}$ ) dominated by sodium and sulfate ions, occurs along the boundary between Minnesota and the Dakotas. Group 4 lakes are represented in this study by Salt Lake. The profundal sediment of Salt Lake is similar to those of group 3 lakes and has been included with them in this discussion. If we treat profundal sediments as a threc-component system (carbonate, organic, and clastic), two trends are followed by Minnesota lakes from an inorganic clastic base (Fig. 8). The first trend, illustrated by group 1 lakes and most high-organic group 2 lakes, involves addition of organic matter. The second trend, illustrated by the remaining group 2 lakes and those of group 3 , involves addition of carbonate to a predominantly inorganic clastic fraction plus $10-20 \%$ organic matter. As mentioned above, two high-organic group 2 lakes (Nokay and Linwood) appear to be transitional.

To test the grouping of lakes, based primarily on water chemistry but subdivided in relation to major sediment components, we ran a $Q$-mode factor analysis on the 25 organic-, carbonate-, and clastic-related variables listed in Table 1 , plus lake area and maximum depth. By this method, similarities among lakes are examined based on the measured variables and coefficients computed for cach lake. These coefficients, called factor loadings or factor scores, are measures of the degree of similarity among lakes (e.g. Harman 1967; Morrison 1967; Sneath and Sokal 1973). So that we could compare percentages, X-ray diffraction peak heights, areas, and depths in the same analysis, all values for each variable were divided by the maximum value of that variable occurring in all 46 lakes (i.e. $x_{i, j} / x_{j \max } ; i=1$ to 46 lakes; $j=1$ to 27 variables). The input matrix, therefore, consisted of 27 converted variables, each with a range of 0 to 1.0 , and 46 observations (lakes). The actual analysis was done using a FORTRAN IV program developed by Ondrick and Srivastava (1970). A total of 10 factors was extracted, although $83 \%$ of the variance in the data was accounted for about equally by the first three factors, after a varimax rotation (Table 4).

Plots of factor loadings for the first three rotated factors are shown in Fig. 16. Lakes with the highest loadings for factor 1 are all high-carbonate group 2 lakes, whereas lakes with low loadings for this factor are those with little or no sedimentary carbonate. Lakes with high loadings for factor 2 are the high-organic group 1 and group 2 lakes, and lakes with high loadings for factor 3 are those lakes of all groups with high proportions of clastic material.

Figure 16A illustrates the degree to which the three lake groups can be segregated by the factor analysis. Group 3 lakes are segregated by their low loadings for factor 2 (organic fraction) coupled with intermcdiate loadings for factor 1 (carbonate fraction). Group 2 lakes exhibit a wide range in loadings for both factors, and a few highorganic group 2 lakes with no sedimentary carbonate overlap the high-organic group 1 lakes, which are clearly segregated from the group 3 lakes.

Figure 16B shows the same organic- and carbonate-enrichment trends as Fig. 8. Lakes of all groups with high loadings for factor 3 (clastic fraction) form a base from which we can see diverging trends toward high and low loadings for factor 1 (car- 
Table 4. Loadings for first threc rotated factors of $Q$-mode factor analysis, 27 variables, 46 lakes.

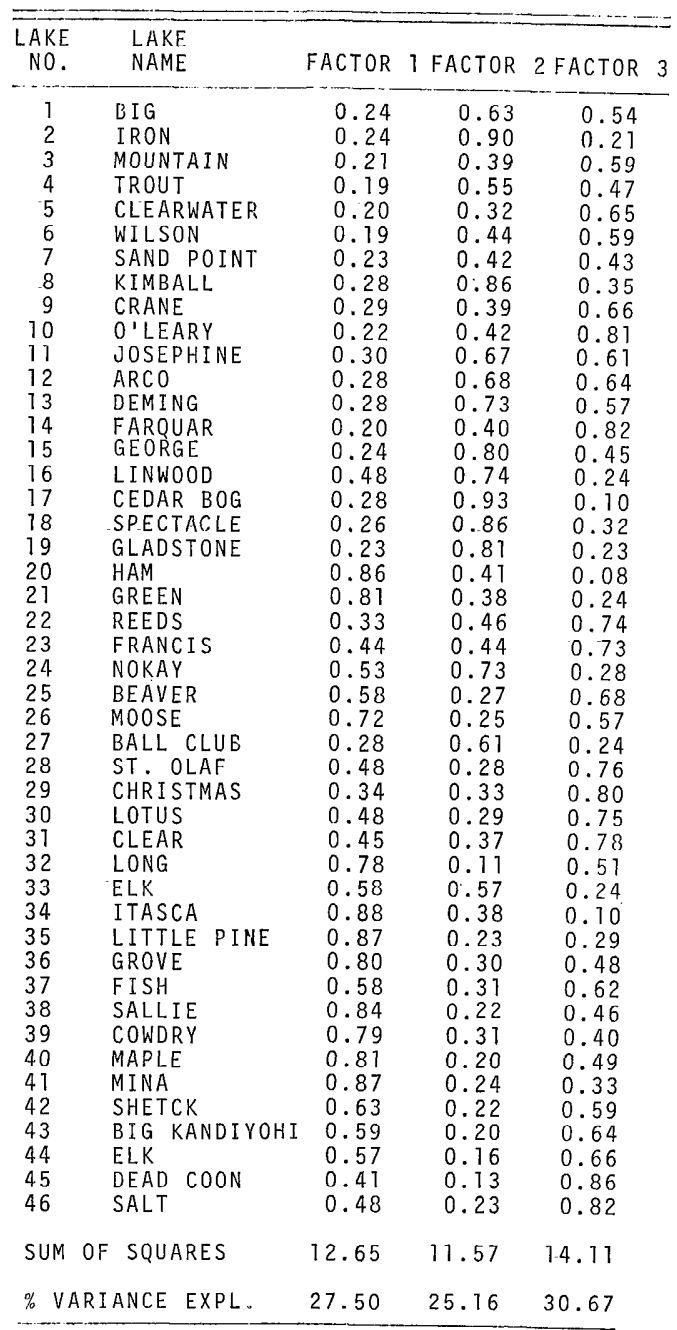

bonate fraction). The lakes with high loadings for factor 1 are the western high-carbonate lakes of group 2 , those with low loadings are the group 1 lakes together with a few group 2 lakes lacking sedimentary carbonate. Three lakes (Linwood, Nokay, and Elk) appear intermediate with regard to these tronds (cf. Fig. 8). Linwood and Nokay (No. 16 and 24) form a high-organic, intermediate carbonate subgroup of the group 2 lakes, and Elk Lakc (Clearwater Co., No. 33) should probably be placed
MINNESOTA LAKE SEDIMENTS $Q-M O D E$ FACTOR ANALYSIS

27 Variables 46 Lakes
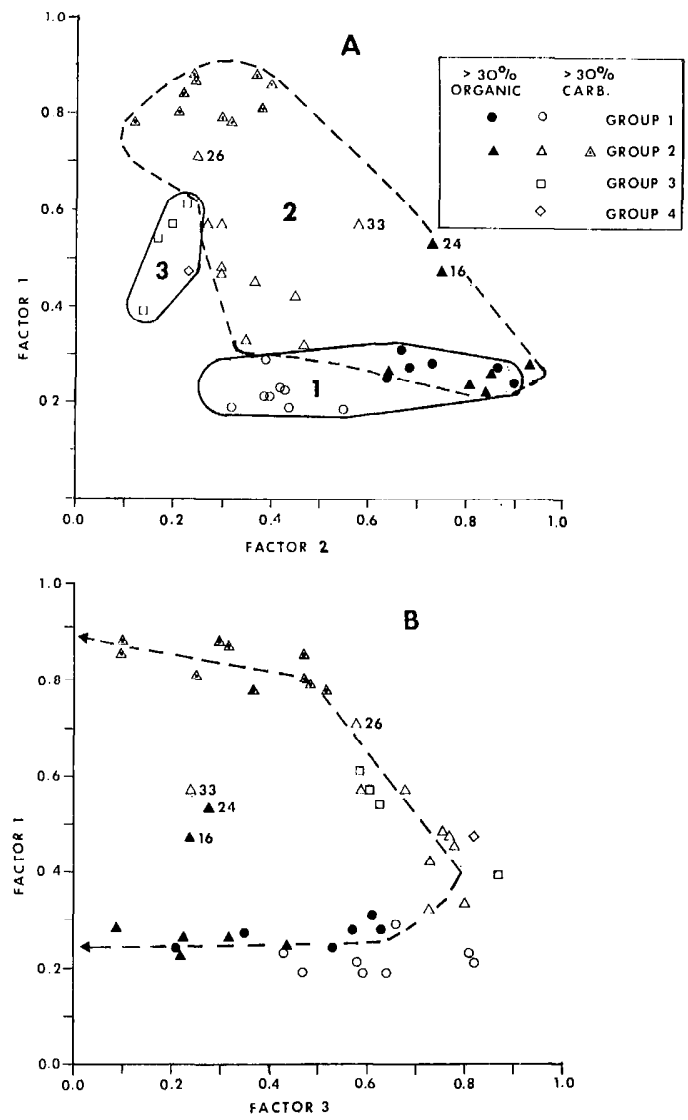

Fig. 16. Scatter plots of rotated factor loadings, $Q$-mode factor analysis of 27 variables in profundal sediments from all 46 lakes. A-Rotated factor 1 vs. rotated factor 2; B-rotated factor 1 vs. rotated factor 3 . Numbers adjacent to points refer to lake numbers in Fig. 2 and Table 4.

with the high-carbonate group 2 lakes, for reasons discussed above.

Associations of variables-Relations among the 27 variables listed in Table 1 are expressed as a correlation matrix in Table 5. To simplify analysis of these relationships, the correlation matrix was used as input for $R$-mode factor analysis using the FORTRAN IV program developed by Ondrick and Srivastava (1970). Results of the $R$ mode factor analysis from the Ondrick and Srivastava program were checked using another factor analysis program (BMD X72) 
(Dixon 1970). Results of both factor analyses were almost identical. Loadings for 10 factors from the Ondrick and Srivastava program, after varimax rotation, are given in Table 6. Each of the first two factors accounts for over $20 \%$ of the variance in the data. The highest loadings for factor 1 are for organic-related variables: loss on ignition at $550^{\circ} \mathrm{C}$, total carbon, organic carbon, hydrogen, and nitrogen. The highest loadings for factor 2 are for carbonate-rclated variables: calcium carbonate calculated from loss on ignition between $550^{\circ}$ and $1,000^{\circ} \mathrm{C}$, soluble $\mathrm{Ca}, \mathrm{X}$-ray calcite peak height, and, to a lesser extent, soluble Mg.

The clastic variables contribute variance to both factor 1 (organic) and factor 2 (carbonate), but are inversoly related to the principal variables in cach factor. This reflects the negative correlation between percent clastic material and both percent carbonate and percent organic matter ( $\mathrm{Ta}$ ble 5 ) and also the two trends (Fig. 8, Fig. 16B) toward either organic or carbonate enrichment of a clastic base. Tables 5 and 6 also show that the inverse relationship bctween carbonate and clastic material is closer than the inverse rolationship between organic matter and clastic material.

The clay-mincral variables of the clastic fraction, illite, chlorite + kaolinite, and clay-size quartz, are negatively corrclated with organic matter but not with carbonate. On the other hand, the total percentage of clastic material, and its major component variables, percent silt and percent clay, do show a negative corrclation with the carbonate fraction. We speculate that this anomaly can be explaincd by considering the geographic distribution of lakes exhibiting these tronds. Most high-organic lakes (groups 1 and 2) are located in north-central and northeastern Minnesota (Fig. 2). In these forested regions, a relativcly thick, moist, acid humus has developed, so that most of the clastic material transported to the lakes is highly weathered and low in total volume. Dilution of this clastic basc with organic mattcr, both accumulating at a relatively slow rate, will result in an inverse relationship between the organic frac- tion and the highly altered clays of the clastic fraction. The carbonatc-rich lakcs, on the other hand, occupy shallow basins in the arid western and southwestern prairie regions. Thorc, the soil cover is not well developed, and erosion is therefore likely to be more rapid. As a result these lakes reccive a greater volume of clastic matorial which, because of increased rate of erosion and low available moisture, is probably less altered by decomposition. Dilution of this clastic base with $\mathrm{CaCO}_{3}$ at a relatively rapid rate results in an inverse relationship bctwecn carbonate and clastic material, most of which is relatively unaltered silt and clay.

The difference in degree of decomposition of clastic materials in the northeastern and central lakes, relative to the southwestcrn lakes, is suggested by the degrec of crystallinity of the clay minerals. The crystallinity index given in Table 3 is much higher for clays from the southwestcrn group 3 lakes than for the northeastern and central lake sediments. This increase in degree of crystallinity is not related to the amount of clay in the sediments or to the total amount of clastic material in the sediments. Gorham et al. (in prep.), in their study of water chemistry of Minnesota lakes, found that the boundary between group 2 and group 3 lakes is not gradual, as might be expected by increascd aridity alone. They conclude that the sharp boundary is the result of differences in glacial drift composition supcrimposed on the regional climatic gradient. The sharp increase in clay crystallinity in group 3 lake scdiments is probably related, therefore, to drift composition as well as to climate.

Factor 3 , accounting for $10 \%$ of the variance in the data, is essentially a clay mineralogy factor. The fact that no other clastic variables have high loadings in this factor suggests that the mineralogy of the clay-size material is independent of the total amount of clastic material being introduced into the lakes.

One might cxpect pronounced regional differences in the clay mineralogy of the profundal sediments, because bedrock types 


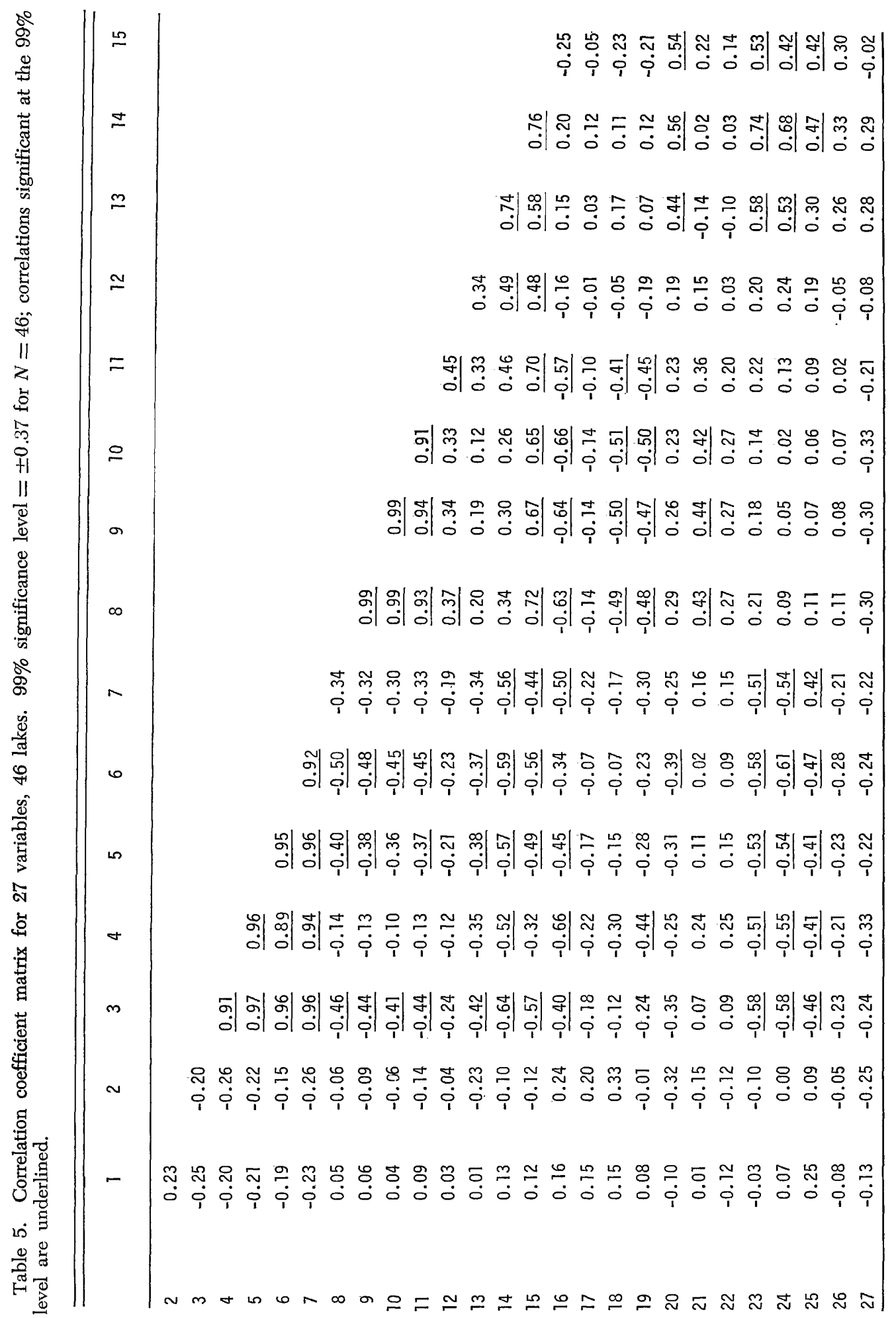




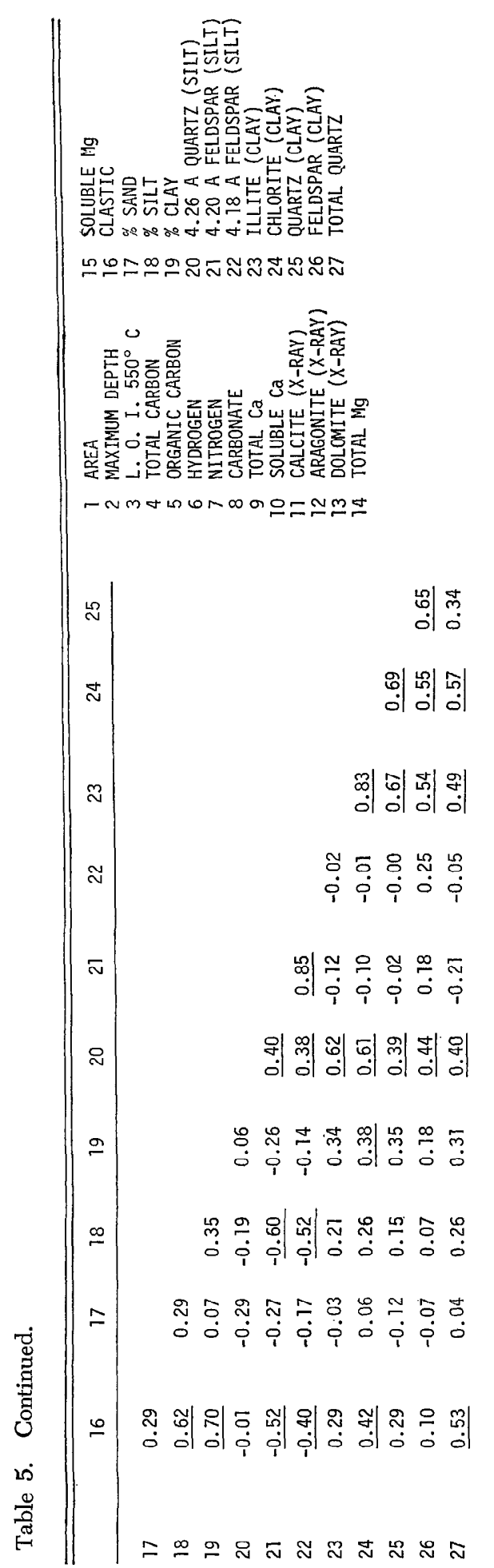

and associated glacial drift vary considerably across the state (Fig. 1). Prccambrian metamorphic and igneous rocks predominate in the northeastern and central parts, Cretaceous shale dominates the lithology in the west, and Paleozoic sandstone and dolomite are dominant in the southeast. The abscnce of marked regional differences suggests that wcathering, as controlled by temperature, available moisture, soil $\mathrm{pH}$, ctc., has a greater influence on clay mineralogy than bedrock type. Pluth et al. (1970) also report a general similarity in mineralogy of the $<5-\mu$ size fraction of 16 soils throughout Minnesota.

However, a hint of bedrock control on clay mineralogy is suggested by factor 10 , which accounts for $10.3 \%$ of the variance in the data. Factors 10 and 2 suggest three contributions of $\mathrm{Mg}$ to lake sediments. In factor 2, soluble $\mathrm{Mg}$ is related to the carbonate variables, indicating that most $\mathrm{Mg}$ soluble in $1.0 \mathrm{~N}$ ammonium acetate is apparcntly derived from $\mathbf{M g}$ coprecipitated with calcite. In factor 10 , however, the highest loadings are for total $\mathrm{Mg}$ and X-ray dolomite. This association indicates that in the western and southwestern lakes, which are high in sedimentary $\mathrm{Mg}$, much of it is tied up as dolomite which is not dissolved by ammonium acetate. A third contribution of $\mathrm{Mg}$ indicated by factor 10 is from clay minerals. Table 5 shows that total $\mathrm{Mg}$ has high positive correlations with both illite and chlorite. Illite is derived mainly from the alteration of potassium feldspar and muscovite, both abundant minerals in acid igneous and metamorphic rocks. Most chlorite is derived from pre-existing metamorphic rocks (e.g. chlorite schist) and some sedimentary rocks. It is a common detrital mineral especially in areas wherc metamorphic rocks have been glaciated (Grim 1953). Consequently chlorite would be expected to be a major clay mineral in Minnesota soils and lake sediments and a major source of $\mathrm{Mg}$ in the sediments. Data from Pluth et al. (1970) show that chlorite is common in soils throughout Minnesota, but is most abundant in the northeastern and cast-central parts underlain by meta- 


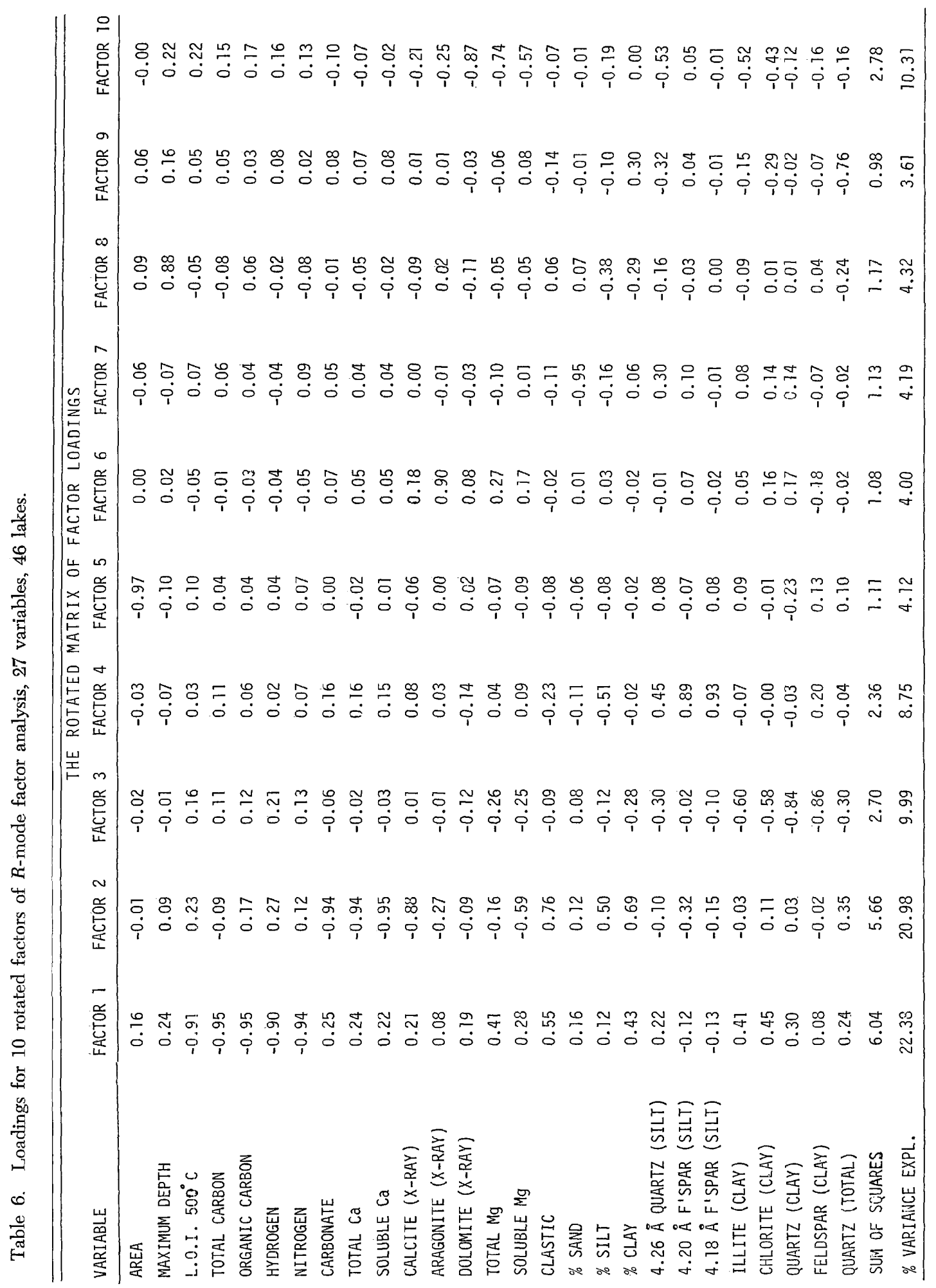


morphic rocks. Group 1 sediments contain up to $1.0 \%$ total $\mathrm{Mg}$, most of which is not soluble in $1.0 \mathrm{~N}$ ammonium acetate (Fig. 12). Most of the $\mathrm{Mg}$ in these sediments is probably tied up in clay mineral structures, particularly in chlorite. This is in agreemont with Callender's (1969) conclusion that most of the Mg in sediments from Lake Supcrior and Lake Michigan not accounted for by calcite and dolomite is ticd up in chloritc. Brunskill ct al. (1971), from their study of sediments in lakes in the Experimental Lakes Area, northwestern Ontario, also conclude that $\mathrm{Mg}$ is not very mobile and tends to remain in sediments.

Another potentially useful index of the degree of weathering is the ratio of quartz to feldspar in a particular size fraction. Because all feldspars are considerably less durable, chemically and physically, than quartz, incrcased weathering should increase quartz relative to feldspar. With an increased rate of erosion and a decreascd degree of weathering, one might expect both the amount of feldspar and the percentage of silt, relative to quartz, to increase with a consequent positive correlation bctween percent silt and feldspar peak height. IIowever, from Tables 5 and 6 it appears that this is not so for the silt-size fraction of these lake sediments. The loadings for factor 4 , which accounts for $8.7 \%$ of the variance in the data, reflect an inverse rolationship between the porcentage of silt and feldspar peak heights in X-ray diffractograms of silt-size material. This apparent contradiction may be explained by obscrving the geographic pattern of silt-size mincralogy. The ratio of the $4.26 \AA$ quartz pcak to the $3.18 \AA$ feldspar peak in silt-size material is mapped in Fig. 15. In the northeastern group 1 lakes, the quartz : feldspar pcak height ratio is generally less than 1.0. However, in the southwestern group 3 lakes, this ratio is gencrally greater than 1.0. The Precambrian crystalline igneous and metamorphic rocks of northeastern Minnesota would be expected to contain relatively high proportions of both plagioclase and orthoclase. The Cretaceous shales of southwestern Minnesota are themsclves weathering products and should therefore contain much lower proportions of feldspars relative to quartz and clay mincrals. Therefore, even though chemical weathering is more intense in the northcast, decomposing feldspars at a faster rate, the weathering products that accumulate as silt in the lakes are enriched in feldspars relative to the less decomposcd clastic debris in the southwest. Whereas weathering has more control than bedrock lithology on clay-size mineralogy, bedrock type appears to be the dominant control on silt-size mincralogy. Presumably this difference is a result of the much larger ratio of surface area to volume in clay-size matcrial, which would make it more vulnerable to decomposition than the silt fractions.

The five rotated factors discussed above account for $72.4 \%$ of the total variance in the data and incorporate most of the relationships among variables. Of the remaining five factors, each explains only about $4 \%$ of the total variance and is the result of a single variable. The main contributing variables in each of these five remaining factors are: lake arca for factor 5, X-ray aragonite for factor 6 , percent sand for factor 7, maximum depth for factor 8 , and $\mathrm{X}$-ray quartz for factor 9 . It is surprising that lake area and depth do not correlate appreciably with any sedimentary variables (Table 5). As discussed above, the main control exerted by depth on mineralogy is its effect on the dissolution of calcite. Howcver, most calcitc-precipitating lakes in Minnesota are sufficiently shallow that dissolution is probably minor. Table 5 shows a slight negative correlation between maximum depth and organic matter but it is not significant at the $99 \%$ level. A hint of depth control on amount of sedimentary organic matter is given by the fact that all deep lakes $(>30 \mathrm{~m})$ contain less than $20 \%$ organic matter in their profundal sediments. However, for lakes shallower than $30 \mathrm{~m}$, there is no relationship between depth and sedimentary organic matter.

Comparison with other lakes-The highorganic group 1 and group 2 lakes are the only Minnesota lakes that have organic con- 
Table 7. Loss on ignition, organic carbon, total nitrogen, organic-C : $\mathrm{N}$ ratio, and carbonate in Minnesota lake sediments compared with other lake groups. All values are based on weight percent dry weight.

\begin{tabular}{|c|c|c|c|c|c|c|}
\hline & $\begin{array}{l}\text { Loss on } \\
\text { Ignition }\end{array}$ & $\begin{array}{l}\text { Organic } \\
\text { Carbon }\end{array}$ & $\begin{array}{l}\text { Total } \\
\text { Nitrogen }\end{array}$ & $\frac{\text { Organic-c }}{\text { Nitrogen }}$ & Carbonate & Reference \\
\hline \multicolumn{7}{|l|}{ MINNESOTA LAKES: } \\
\hline $\begin{aligned} & 8 \text { Low-Org. G } 1 \\
& 13 \text { High-0rg. G } 1 \& 2 \\
& 10 \text { Intermed. G } 2 \\
& 10 \text { High-Carb. G } 2 \\
& 5 \text { Group } 3 \\
& 46 \text { Total }\end{aligned}$ & $\begin{array}{l}18 \pm 2 * \\
42 \pm 7 \\
18 \pm 6 \\
20 \pm 4 \\
12 \pm 4 \\
25 \pm 12\end{array}$ & $\begin{aligned} 8 & \pm 1 \\
21 & \pm 4 \\
9 & \pm 2 \\
10 & \pm 2 \\
6 & \pm 3 \\
12 & \pm 6\end{aligned}$ & $\begin{array}{l}0.8 \pm 0.2 \\
2.4 \pm 0.5 \\
1.1 \pm 0.3 \\
1.2 \pm 0.2 \\
0.6 \pm 0.3 \\
1.4 \pm 0.7\end{array}$ & $\begin{array}{l}9.6 \\
8.9 \\
8.7 \\
8.5 \\
9.5 \\
8.9\end{array}$ & $\begin{array}{r}\text { none } \\
\text { none } \\
17 \pm 7 \\
39 \pm 6 \\
20 \pm 2\end{array}$ & \\
\hline $\begin{array}{l}\text { 16 Precambrian } \\
\text { Shield Lakes, } \\
\text { Experimental Lakes } \\
\text { Area, N.W. Ontario }\end{array}$ & $44 \pm 14$ & $20 \pm 7$ & $2.1 \pm 0.8$ & 9.7 & none & $\begin{array}{l}\text { Brunski11 } \\
\text { et a1. } \\
1971\end{array}$ \\
\hline \multicolumn{7}{|l|}{ GREAT LAKES: } \\
\hline $\begin{array}{l}\text { Ontario } \\
\text { Erie }^{+} \\
\text {Huron } \\
\text { South. Michigan } \\
\text { Central Michigan } \\
\text { North. Michigan } \\
\text { Green Bay } \\
\text { Superior }\end{array}$ & & $\begin{array}{c}3.5 \\
2.5 \\
2.7 \\
1.6 \pm 0.7 \\
1.0+0.9 \\
1.7 \pm 0.8 \\
2.6 \pm 1.4 \\
2.4\end{array}$ & $\begin{array}{c}0.39 \\
0.33 \\
0.39 \\
0.20 \pm 0.09 \\
0.18 \pm 0.11 \\
0.24 \pm 0.11 \\
0.31 \pm 0.20 \\
0.23\end{array}$ & $\begin{array}{r}8.2 \\
8.5 \\
7.7 \\
7.6 \\
5.5 \\
7.1 \\
8.4 \\
10.6\end{array}$ & $\begin{array}{c}5.0^{\ddagger} \\
5.9^{\ddagger} \\
5.8 \\
8.5 \pm 4.2 \\
6.4 \pm 4.6 \\
8.8 \pm 4.6 \\
4.7 \pm 3.3 \\
1.14\end{array}$ & $\begin{array}{l}\text { Kemp "1971 } \\
" \text { " } \\
\text { Callender } \\
1969 " \\
" \text { " } \\
"\end{array}$ \\
\hline \multicolumn{7}{|l|}{ WISCONSIN LAKES: } \\
\hline $\begin{array}{l}\text { Mendota } \\
\text { La Belle } \\
23 \text { Wisc. Lakes }\end{array}$ & 46 & $\begin{array}{c}7.3 \\
7.3 \\
20 \pm 10\end{array}$ & $\begin{array}{c}0.93 \\
0.65 \\
1.8 \pm 0.7 \text { 5 }\end{array}$ & $\begin{array}{r}7.8 \\
11.1 \\
11.0\end{array}$ & $\begin{array}{ll} & 35 \\
& 45 \\
\text { not } & \text { given }\end{array}$ & $\begin{array}{l}\text { Konrad et } \\
\text { al. 1970 } \\
\text { Compiled by } \\
\text { Brunskill } \\
\text { et al. } 1971\end{array}$ \\
\hline \multicolumn{7}{|l|}{ ENGLISH LAKES: } \\
\hline $\begin{array}{l}5 \text { Group } 1 \| \\
5 \text { Group } 2 \\
6 \text { Group } 3 \\
16 \text { Total }\end{array}$ & $\begin{array}{l}14.6 \\
16.2 \\
17.0 \\
16.0\end{array}$ & $\begin{array}{l}6.1 \\
7.1 \\
7.8 \\
7.0\end{array}$ & $\begin{array}{l}0.49 \\
0.57 \\
0.65 \\
0.58\end{array}$ & $\begin{array}{l}12.0 \\
13.0 \\
12.0 \\
12.3\end{array}$ & $\begin{array}{l}\text { none } \\
\text { none } \\
\text { none } \\
\text { none }\end{array}$ & $\begin{array}{l}\text { Gorham et } \\
\text { al. } 1974\end{array}$ \\
\hline
\end{tabular}

* A11 \pm values represent one standard deviation.

+ Means of basin samples only; 50 samples for Ontario and 59 samples for Erie.

$\ddagger$ Calculated from values of carbonate-carbon.

§ Kjeldaht Nitrogen.

II Group 1 (least productive) includes Wastwater, Thirlmere, Buttermere, Ennerdale, and Crummock; Group 2 (intermediate productivity) includes Haweswater, Coniston, Rydal, Windermere North Basin, and Derwentwater; Group 3 (most productive) includes Loweswater, Windermere South Basin, Blelham Tarn, Ul1swater, Bassenthwaite, and Esthwaite.

tents in their profundal sediments even approaching those of lakes in Wisconsin and the Experimental Lakes Area (ELA) of Ontario (Table 7). Most of those lakes resemble group 1 lakes in their water chemistry. As Brunskill et al. (1971) point out, the high organic content of profundal sediments in the EI_A lakes results not from high primary production, but from lack of dilution by clastic material and an abundance of organic matter in soils of the drainage basin. Lack of correlation between organic productivity and sedimentary organic matter is also noted by Kleerekoper and Grenier (1952) for Lake Lauzon, a deep lake on the Precambrian shield of Quebec. 
Although Lake Lauzon is oligotrophic, profundal sediments contain up to $75 \%$ organic matter (average $45.6 \%$ ), attributed to inhibition of organic decomposition. Gorham (1958) found that sediments from 54 dilute and generally unproductive Scottish lakes had ignition loss values ranging from 4$70 \%$ with a median of only $20 \%$. Those lakes, however, are in a mountainous region favorable to erosion of clastic material.

Brunskill et al. (1971) concluded that most organic material in the ELA lakes is derived from their drainage basins. A similar conclusion was reached by Mackereth (1966) for organic matter in profundal sediments from the English lakes. Data on sedimentary pigments suggest that an appreciable part of the sedimentary organic matter in deep unproductive group 1 lakes in northeastern Minnesota was probably derived from soils in the drainage basins (Sanger and Gorham 1970; Gorham and Sanger 1975). However, in the more productive of the English lakes, as in the more productive Minnesota lakes, pigment data (Gorham 1960; Gorham and Sanger 1967, 1975; Gorham ct al. 1974; Sanger and Gorham 1970) demonstrate clearly that most organic matter is derived from primary production within the lakes.

The profundal sediments of the low-organic group 1 lakes are most like those of the English lakes insofar as they generally contain less than $20 \%$ organic matter. They are not nearly so low in organic content as profundal sediments from the Great Lakes. The main difference between sediments from the low-organic group 1 Minnesota lakes and the English lakes is the higher $C: N$ ratio of the English sediments. Because both the English and Minnesota samples were analyzed in the same laboratory at about the same time, there is little chance that this difference can be attributed to analytical technique. The higher $C: N$ ratio in the English lake sediments may mean that on average these lakes receive a greater input of more resistant soil organic matter, rich in carbon, than the Minnesota lakes. In general, differences of $C: N$ ratio in pro- fundal sediments are not very great, with an overall range of 5.5-13.0 (Table 7).

The high-carbonate profundal sediments from the western Minnesota lakes are clearly distinguishable from those of most other lake groups listed in Table 7, except the high-carbonate Wisconsin lakes, Mendota and LaBelle.

\section{References}

Acknoyd, E. A., W. C. Walton, and D. L. Hillus. 1967. Ground-water contribution to stream flow and its relation to basin characteristics in Minnesota. Minn. Geol. Surv. Rep. Invest. 6.36 p.

Baken, D. G., AND J. HI. Strub. 1965. Climate of Minnesota, Part 3. Univ. Minn. Agr. Exp. Sta. Tech. Bull. 248. 12 p.

Boone, G. M., AND E. P. WheELER. 1968 Staining for cordierite in thin section. Am. Mineral. 53 : $327-331$.

Bright, R. C. 1968. Surface water chemistry of some Minnesota lakes, with preliminary notes on diatoms. Univ. Minn. Limnol. Res. Center, Interim Rep. 3. 58 p.

Brunskill, G. J., D. Povoledo, B. W. Graham, AND M. P. Stainton. 1971. Chemistry of surface sediments of sixteen lakes in the Experimental Lakes Area, northwestern Ontario. J. Fish. Res. Bd. Can. 28: 277-294.

Callender, E. 1969. Geochemical characteristics of Lakes Michigan and Superior sediments. Proc. 12th Conf. Great Lakes Res. 1969: 124-160.

Carnoll, D. 1970. Clay minerals: A guide to their X-ray identification. Geol. Soc. $\Lambda \mathrm{m}$. Spec. Pap. 126. 80 p.

Dean, W. E., Jr. 1974. Determination of carbonate and organic matter in calcareous sediments and sedimentary rocks by loss on ignition: Comparison with other methods. J. Sediment. Petrol. 44: 242-248.

Dixon, W. J. [Ed.]. 1970. BMD Biomedical computer programs, X-serics supplement. Univ. Calif. Publ. Automatic Computation 2 (2nd ed.). 260 p.

Gorham, E. 1958. The physical limnology of northern Britain: An epitome of the bathymetrical survey of the Scottish freshwater lochs, 1897-1909. Limnol. Oceanogr. 3 : 4050.

- 1960. Chlorophyll derivatives in surface muds from the English lakes. Limnol. Occanogr. 5: 29-33.

—, J. W. G. LUND, J. E. SANGer, AND W. E. DEAN, JR. 1974. Some relationships between algal standing crop, water chemistry, and sediment chemistry in the English lakes. Limnol. Oceanogr. 19: 601-617. 
-, ANग J. E. SANGER. 1967. Plant pigments in woodland soils. Ecology 48: 306-308.

, $\Lambda N D \longrightarrow$ 1975. Fossil pigments in Minncsota lake scdiments, and their bearing upon balance between terrestrial and aquatic inputs to sedimentary organic matter. Int. Ver. Theor. Angow. Limnol. Verh. 19: in press.

Grim, R. E. 1953, Clay mincralogy. McGrawIlill.

II $\mathbf{R M A N}$, II. II. 1967. Modern factor analysis, 2nd ed. Chicago.

IIUTChinson, G. E. 1957. Treatise on limnology, v. 1. Wilcy.

Jackson, M. L. 1967. Soil chemical analysisadvanced coursc. M. L. Jackson, Dcp. Soil. Sci., Univ. Wisconsin, Madison. 894 p.

KEMP, A. L. W. 1971. Organic carbon and nitrogen in the surface sediments of Lake Ontario, Erie, and IIuron. J. Scdiment. Petrol. 41: $537-548$.

Klefrekoper, II., and F. Grenier. 1952. The bottom sediments of Lake Lauzon, Montcalm County, Province of Quebec, 1. Chemical and physical investigations. Can. J. Zool. 30: 219-242.

Konrad, J. G., D. R. Keeney, G. Chesters, and K. Cinen. 1970. Nitrogen and carbon distribution in sediment cores of selected Wisconsin lakes. J. Water Pollut. Control Fed. 42: 2094-2101.

MACkeneth, T. J. II. 1966. Some chemical observations on post-glacial lake sediments. Phil. Trans. R. Soc. I ond. Sor. B 250: 165213.

Merami, R. P. 1968. Planktonic photosynthesis and the environment of carbonate deposition in lakes. Univ. Minn. Limnol. Res. Center, Interim Rep. 2. 47 p.

Minnesota Department of Conservation. 1962. Water resources of Minnesota, a study guide. Minn. Dep. Conserv. Div. Waters Bull. 16. 28 p.

Mrtien, II. T., C. II. Scott, ANd P. G. Rosene. 1968. Chemical quality of surface waters in Devils' Lake Basin, North Dakota, 1952-60.
U.S. Gcol. Surv. Water Supply Pap. 1859-8. $42 \mathrm{p}$.

Morrison, J. F. 1967. Multivariate statistical methods. McGraw-Hill.

Montimin, C. II. 1941. The exchange of dissolved substances between mud and water in lakes. J. Ecol. 29: 280-329.

- - 1942. The exchange of dissolved substances between mud and water in lakes. J. Ecol. 30: 147-201.

Mül.t.fr, G., G. Irion, And U. Förstner. 1972. Formation and diagenesis of inorganic $\mathrm{Ca}-\mathrm{Mg}$ carbonates in the lacustrine environment. Naturwissenschaften 59: 158-164.

Onidick, C. W., and G. S. Srivastava. 1970. CORFAN-FORTRAN IV computer program for correlation, factor analysis ( $R$ - and Qmode) and varimax rotation. Kansas Geol. Surv. Computer Contrib. 42.

Plutit, D. J., R. S. Adams, JR., R. II. Rust, and J. R. Perenson. 1970. Characteristics of selected horizons from 16 soil series in Minnesota. Univ. Minn. Agr. Exp. Sta. Tech. Bull. 272. $34 \mathrm{p}$.

Ross, C. S., Ann P. F. KenR. 1931. The clay minerals and their identification. J. Sediment. Petrol. 1 : 55-65.

SAnger, J. E., AND E. Gorham. 1970. The diversity of pigments in lake sediments and its ccological significance. Limnol. Occanogr. 15: 59-69.

Sims, P. K., AND G. B. Morey. 1972. Gcology of Minnesota: A centennial volume. Minn. Geol. Surv. 632 p.

Sneatti, P. FI. A., and R. R. Sokal. 1973. Numerical taxonomy, the principles and practice of numcrical classification. Frecman.

WANGERSKY, P. J, AND O. I. JoensuU. 1967. The fractionation of carbonate in decp-sea cores. J. Gool. 75: 148-177.

Winter, T. C. 1974. The natural quality of ground water in Minnesota. Minn. Dep. Nat. Resour., Div. Waters, Soils, Minerals. Bull. 26. $25 \mathrm{p}$.

Submitted: 13 January 1975 Accepted: 24 November 1975 